\title{
ARE DUSTY GALAXIES BLUE? INSIGHTS ON UV ATTENUATION FROM DUST-SELECTED GALAXIES
}

\author{
C. M. Casey ${ }^{1}$, N. Z. Scoville ${ }^{2}$, D. B. SAnders ${ }^{3}$, N. LeE $^{3}$, A. Cooray ${ }^{1}$, S. L. FinKelstein ${ }^{4}$, P. CAPak ${ }^{5}$, A. Conley ${ }^{6}$,

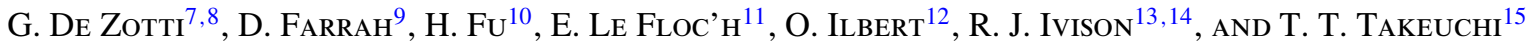 \\ ${ }^{1}$ Department of Physics and Astronomy, University of California, Irvine, Irvine, CA 92697, USA \\ ${ }^{2}$ California Institute of Technology, 1216 East California Boulevard, Pasadena, CA 91125, USA \\ ${ }^{3}$ Institute for Astronomy, University of Hawai'i, 2680 Woodlawn Drive, Honolulu, HI 96822, USA \\ ${ }^{4}$ Department of Astronomy, The University of Texas at Austin, Austin, TX 78712, USA \\ ${ }^{5}$ Spitzer Science Center, California Institute of Technology, 1200 East California Boulevard, Pasadena, CA 91125, USA \\ ${ }^{6}$ Center for Astrophysics and Space Astronomy 389-UCB, University of Colorado, Boulder, CO 80309, USA \\ ${ }^{7}$ Osservatorio Astronomico di Padova, Vicolo dell'Osservatorio 2, I-35122 Padova, Italy \\ ${ }^{8}$ SISSA, Via Bonomea 265, I-34136 Trieste, Italy \\ ${ }^{9}$ Department of Physics, Virginia Tech, Blacksburg, VA 24061, USA \\ ${ }^{10}$ Department of Physics and Astronomy, University of Iowa, Iowa City, IA 52242, USA \\ 11 CEA-Saclay, Orme des Merisiers, bât. 709, F-91191 Gif-sur-Yvette Cedex, France \\ 12 Aix Marseille Université, CNRS, Laboratoire d'Astrophysique de marseille, UMR 7326, F-13388 Marseille, France \\ ${ }^{13}$ Institute for Astronomy, University of Edinburgh, Royal Observatory, Blackford Hill, Edinburgh, EH9 3HJ, UK \\ ${ }^{14}$ European Southern Observatory, Karl-Schwarzschild-Strasse 2, D-85748 Garching bei München, Germany \\ ${ }^{15}$ Nagoya University, Division of Particle and Astrophysical Science, Furo-cho, Chikusa-ku, Nagoya 464-8602, Japan \\ Received 2014 June 23; accepted 2014 September 25; published 2014 November 12
}

\begin{abstract}
Galaxies' rest-frame ultraviolet (UV) properties are often used to directly infer the degree to which dust obscuration affects the measurement of star formation rates (SFRs). While much recent work has focused on calibrating dust attenuation in galaxies selected at rest-frame ultraviolet wavelengths, locally and at high- $z$, here we investigate attenuation in dusty, star forming galaxies (DSFGs) selected at far-infrared wavelengths. By combining multiwavelength coverage across $0.15-500 \mu \mathrm{m}$ in the COSMOS field, in particular making use of Herschel imaging, and a rich data set on local galaxies, we find an empirical variation in the relationship between the rest-frame UV slope $(\beta)$ and the ratio of infrared-to-ultraviolet emission $\left(L_{\mathrm{IR}} / L_{\mathrm{UV}} \equiv \mathrm{IRX}\right)$ as a function of infrared luminosity, or total SFR. Both locally and at high $-z$, galaxies above SFR $\gtrsim 50 M_{\odot} \mathrm{yr}^{-1}$ deviate from the nominal IRX- $\beta$ relation toward bluer colors by a factor proportional to their increasing IR luminosity. We also estimate contamination rates of DSFGs on high- $z$ dropout searches of $\ll 1 \%$ at $z \lesssim 4-10$, providing independent verification that contamination from very dusty foreground galaxies is low in Lyman-break galaxy searches. Overall, our results are consistent with the physical interpretation that DSFGs, e.g., galaxies with $>50 M_{\odot} \mathrm{yr}^{-1}$, are dominated at all epochs by short-lived, extreme burst events, producing many young $\mathrm{O}$ and B stars that are primarily, yet not entirely, enshrouded in thick dust cocoons. The blue rest-frame UV slopes of DSFGs are inconsistent with the suggestion that most DSFGs at $z \sim 2$ exhibit steady-state star formation in secular disks.
\end{abstract}

Key words: galaxies: evolution - galaxies: high-redshift - galaxies: starburst - infrared: galaxies submillimeter: galaxies

Online-only material: color figures

\section{INTRODUCTION}

A key goal of extragalactic astrophysics is the accurate census and measurement of the cosmic star formation rate density (SFRD) across cosmic time. The first measurements of the SFRD (Tinsley \& Danly 1980; Lilly et al. 1995; Madau et al. 1996) revealed that the density of star formation has decreased 10 -fold over the last seven billion years since $z \sim 1$. More recent measurements (like those summarized in Hopkins \& Beacom 2006) show the nature of the SFRD at the highest redshifts, reaching a plateau at $z \sim 2$, and steadily declining at earlier times (now extended to $z \sim 10$ with very deep near-infrared data, e.g., Oesch et al. 2013). However, there is one major caveat to SFRD measurements derived from direct starlight in the ultraviolet, optical, and near-infrared, and that is the effect of dust obscuration.

Detailed studies of dust attenuation in nearby star forming regions and star forming galaxies (e.g., Calzetti et al. 1994; Calzetti 2001; Meurer et al. 1999; Overzier et al. 2011) have long served as a calibration tool for understanding infrared reprocessed emission in galaxies out to high redshift. One critical tool has been the empirically observed tight correlation between galaxies' rest-frame ultraviolet (UV) slope, defined as $\beta$, and the ratio of infrared luminosity to UV luminosity at $\approx 1600 \AA$, defined as IRX $\equiv L_{\mathrm{IR}} / L_{\mathrm{UV}}$. This local relationship between $\beta$ and IRX has been widely used as a method to infer dust obscuration, and thus total star formation rates (SFRs), in high-redshift galaxies in the absence of far-infrared data (e.g., Bouwens et al. 2009). Due to the substantial recent development in deep, far-infrared instrumentation (see the review of Casey et al. 2014 for a complete discussion of far-infrared data sets used at high redshift), the robustness of this IRX $-\beta$ relationship can now be tested at high redshifts and high luminosities.

Until recently, the analysis of the rest-frame UV properties of dusty galaxies was limited to small, inhomogeneous samples. Here we combine the strengths of recent large area far-infrared surveys from Herschel with the extensive $2 \mathrm{deg}^{2} 30+$ band UV/optical/near-infrared photometry in the COSMOS field (Capak et al. 2007; Scoville et al. 2007, 2013) to investigate the rest-frame UV characteristics of large samples of high- $z$ dusty star forming galaxies (DSFGs). We compare the analysis of the $z>0$ DSFGs to data of local galaxies of all luminosities 
(Gil de Paz et al. 2007; Howell et al. 2010; U et al. 2012) to investigate the underlying physical characteristics of dust attenuation at both low and high redshifts. Section 2 describes some of the relevant history of the IRX- $\beta$ relation, and Section 3 describes our galaxy samples-both nearby and at $z>0$ in the COSMOS field - as well as presenting basic calculations. Our analysis of DSFGs in the IRX- $\beta$ plane is presented in Section 4. We address the possible contamination of high- $z$ Lyman-break galaxy (LBG) dropout studies in Section 5. Our discussion is later presented in Section 6 and conclusions in Section 7. Throughout, we assume a Salpeter initial mass function (IMF; Salpeter 1955, although we note conversion to a different IMF, thus different SFR, is straightforward) and a flat $\Lambda$ CDM cosmology (Hinshaw et al. 2009) with $H_{0}=71 \mathrm{~km} \mathrm{~s}^{-1} \mathrm{Mpc}^{-1}$ and $\Omega_{\mathrm{M}}=0.27$.

\section{THE IRX $-\beta$ RELATION TO DATE}

The relationship between galaxies' relative dust attenuation, measured as the ratio of IR $(8-1000 \mu \mathrm{m})$ to UV (1600 $\AA$ ) luminosity $L_{\mathrm{IR}} / L_{\mathrm{UV}} \equiv \mathrm{IRX}$, and their rest-frame ultraviolet color was first studied in a sample of nearby starburst galaxies (Meurer et al. 1995, 1999). Investigating the ultraviolet emission in nearby galaxies must be done from space, and the first observations to contribute to this area came from the International Ultraviolet Explorer (IUE) satellite (Kinney et al. 1993). While the IUE played a critical role in laying the groundwork for UV analyses of galaxies, and establishing our understanding of the IRX $-\beta$ relationship, a key limitation of IUE data was its small aperture/field of view: $10^{\prime \prime} \times 20^{\prime \prime}$, typically much smaller than the full spatial extent of nearby galaxies (a few arcminutes across). It should not then be surprising that the IUE data of Kinney et al. (1993) was focused on galaxy cores, which led to an underprediction of galaxies' total UV luminosities.

In contrast to the UV, IRAS 12-100 $\mu \mathrm{m}$ observations (Neugebauer et al. 1984) and subsequent far-infrared observations of nearby galaxies (e.g., Dunne et al. 2003; Kawada et al. 2007), have been limited to very large apertures. Without matched apertures in the UV and IR, the original analysis of the IRX- $\beta$ relation in Meurer et al. (1999) was biased by overestimating IRX, even though their selection of blue compact dwarfs attempted to circumvent this problem. Furthermore, the $I U E$ focus on only galaxy cores implied that there could also likely be a UV color bias, with potential underestimation of the global $\beta$ by only pointing toward the blue, UV-bright cores. Due to later observations from the Galaxy Evolution Explorer (GALEX; Morrissey et al. 2007), which provided wide fieldof-view UV photometry for the same nearby galaxies (Gil de Paz et al. 2007), a recent revised analysis of the Meurer et al. relation finds lower values of IRX and redder UV slopes for the exact same galaxies (see Takeuchi et al. 2012, as well as some discussion in Overzier et al. 2011).

Besides differences in photometry, it has long been known that galaxies of different types present differently in the IRX- $\beta$ plane. Young, metal-poor galaxies like the Small Magellanic Cloud (SMC) and Large Magellanic Cloud (LMC) are redder and less dusty than starbursts, and normal galaxies lie between the young SMC-type galaxies and compact blue starbursts (Buat et al. 2005, 2010; Seibert et al. 2005; Cortese et al. 2006; Boissier et al. 2007; Boquien et al. 2009, 2012; Muñoz-Mateos et al. 2009; Takeuchi et al. 2010; Hao et al. 2011; Overzier et al. 2011). Much of these differences are also likely caused by the differences in internal attenuation curve, whether steep, shallow, and with or without the $2175 \AA$ feature (Gordon et al. 2000; Burgarella et al. 2005). Kong et al. (2004) provided a theoretical framework for the interpretation of these differences by parameterizing galaxy types with a birthrate parameter, $b$, defined as the ratio of present to past-averaged SFR, whereby starbursts will have a much higher fraction of FUV emission $(\sim 0.153 \mu \mathrm{m})$ to NUV emission $(\sim 0.231 \mu \mathrm{m})$ from $\mathrm{O}$ and $\mathrm{B}$ stars. While Kong et al. attribute the difference between normal galaxies and starbursts to a difference in star formation histories, Seibert et al. (2005) and Cortese et al. (2006) argue using $G A L E X$ data that the differences are likely due to different dust geometries.

Of particular interest for this paper is the observation that ultraluminous infrared galaxies (ULIRGs) and related IR-bright galaxy populations lie significantly above the IRX $-\beta$ relation, where IRX and $\beta$ have been claimed to be completely uncorrelated (Goldader et al. 2002; Burgarella et al. 2005; Buat et al. 2005; Howell et al. 2010; Takeuchi et al. 2010). Howell et al. (2010) interpret sources lying above the IRX $-\beta$ relation as having an excess of dust, and that the difference in IRX ( $\Delta$ IRX) from the expected relation represents the extent to which the IR and UV emission is decoupled. Boquien et al. (2009) and Muñoz-Mateos et al. (2009) perform detailed studies of nearby galaxies and conclude that both dust geometry and star formation history have a substantial impact on the placement of galaxies on the IRX- $\beta$ relation. Given the heightened importance of dusty ULIRGs to cosmic star formation at $z \sim 1$ and beyond (Le Floc'h et al. 2005; Caputi et al. 2007; Casey et al. $2012 \mathrm{a}, 2012 \mathrm{~b}$ ), it seems crucial to understand IRX $-\beta$ in dusty galaxies as well as unobscured galaxies. To date, this has been unclear.

Beyond the nearby universe, the Meurer et al. (1999) IRX- $\beta$ relation has played a fundamental role in estimating the amount of dust attenuation at high redshifts, particularly at $z>3$, where direct infrared observations are unavailable (Ouchi et al. 2004; Stanway et al. 2005; Hathi et al. 2008; Bouwens et al. 2009). For example, Bouwens et al. (2009) use measurements of the rest-frame UV slope $\beta$ of very faint near-IR-detected galaxies at $6<z_{\text {phot }}<10$ to constrain the dustiness of the early universe. Because Bouwens et al. find that sources in high- $z$ surveys are significantly bluer than low- $z$ galaxies, they conclude that the dust obscuration plays an insignificant role in galaxy evolution at $z>5$. Given that the nominal IRX $-\beta$ relation is actually quite uncertain (and based on potentially biased galaxy samples), this constraint on high- $z$ dust obscuration needs independent verification from infrared/submillimeter surveys.

Constraining dust obscuration (and SFRs) at the highest redshifts requires a thorough understanding and calibration of the IRX $-\beta$ relation beyond the local universe. Unfortunately, calibrations of the IRX $-\beta$ relation have been very limited due to long-standing limitations and sensitivity of far-infrared observations. The most thorough analysis of the IRX $-\beta$ relation at high- $z$ has come from studies of spectroscopically confirmed $z \sim 2$ LBGs (Reddy et al. 2006; Reddy \& Steidel 2009; Reddy et al. 2010, 2012). While earlier works (those before about 2010) largely relied on indirect measurements of galaxies' far-infrared luminosity, Reddy et al. (2012) use some of the deepest pointings of the Herschel Space Observatory in the GOODS fields (Elbaz et al. 2011) to investigate the direct far-infrared emission in LBGs. Since very few LBGs are directly detected with Herschel, they used a stacking analysis to measure the characteristic $L_{\mathrm{IR}}$ for $z \sim 2 \mathrm{LBGs}$ and found $\left\langle L_{\mathrm{IR}}(\mathrm{LBG})\right\rangle \sim 10^{11} L_{\odot}$; this characteristic luminosity indicates 
that $\sim 80 \%$ of the star formation is obscured in $L_{*}$ galaxies at $z \sim 2$. This lines up with expectation from the nominal Meurer et al. (1999) IRX- $\beta$ attenuation curve (in spite of its known problems caused by IUE aperture limitations and its lack of accommodation for "normal" type galaxies). Recently, work on $z \sim 4$ LBGs (To et al. 2014), using radio continuum measurements instead of direct far-infrared data, show further agreement with the Meurer et al. relation. Similar to the Reddy et al. results, Heinis et al. (2013) explore the dust attenuation law in large samples of UV-selected galaxies at $z \sim 1.5$ and find a roughly constant IRX ratio over a wide range of UV luminosity explored and an IRX $-\beta$ relation in line with local "normal" star forming galaxies. Further stacking results beyond $z>3$ potentially hint at a breakdown in the relation for highly luminous LBGs (Lee et al. 2012; Coppin et al. 2014).

While the Reddy et al. and Heinis et al. direct far-infrared studies have shed valuable light on dust attenuation calibrations at high- $z$, they both address the problem using UV-selected galaxy samples, which preferentially might have bluer UV colors and lower IRX values than the average galaxy at high redshift. In contrast, Penner et al. (2012) present an analysis of the IRX $-\beta$ relationship for $24 \mu \mathrm{m}$ selected dust-obscured galaxies (DOGs; Dey et al. 2008), which have direct detections in the far-infrared from Herschel-PACs (Poglitsch et al. 2010). They find, perhaps not surprisingly, that dustier galaxies have higher $L_{\mathrm{IR}} / L_{\mathrm{UV}}$ ratios than those selected at UV wavelengths, even at matched rest-frame UV slopes. This is quite similar to earlier work on local ULIRGs (Goldader et al. 2002; Howell et al. 2010), which found similarly high $L_{\mathrm{IR}} / L_{\mathrm{UV}}$ ratios, above that expected from IRX- $\beta$. This difference between "normal" star forming galaxies and infrared-luminous starbursts is attributed to emergent UV emission not corresponding to the same spatial regions of dust absorption and re-emission (Trentham et al. 1999; Papovich et al. 2006; Bauer et al. 2011). Indeed, some select studies describing the rest-frame UV and optical properties of submillimeter galaxies (SMGs; selected at $850 \mu \mathrm{m}$; Smail et al. 1997) at high redshift reinforce this result by finding little correspondence between UV luminosity, UV color, and FIR luminosity (Frayer et al. 2000; Smail et al. 2004; Chapman et al. 2005; Casey et al. 2009; Walter et al. 2012; Fu et al. 2012). This evidence has so far indicated that the nominal IRX- $\beta$ relation should not be used in dusty galaxies (and vice versa, that any relationship determined for dusty galaxies should not be assumed for a general galaxy population), but what more can we learn?

\section{GALAXY SAMPLES AND CALCULATIONS}

We use two sets of galaxies in this analysis; the first is a set of nearby galaxies $(z<0.085)$ spanning the characteristic range of galaxy environments in the local volume, with $L_{\text {bol }} \sim 10^{(8-12.5)} L_{\odot}$ and SFRs $\approx 0.01-100 M_{\odot} \mathrm{yr}^{-1}$. The second galaxy population consists of far-infrared-selected star forming galaxies spanning a redshift range $0<z<5$, with most at $z<3.5$. The selection, data sets, and description of these samples follow. The calculation of relevant quantities like $L_{\mathrm{UV}}, L_{\mathrm{IR}}$, IRX, and $\beta$ are performed in a consistent manner between local and $z>0$ samples.

\subsection{Nearby Galaxies}

Our local data set combines the 1034 nearby galaxies observed by GALEX included in Gil de Paz et al. (2007), originally selected from the GALEX Nearby Galaxies Survey, and the 202 nearby GOALS (Armus et al. 2009) LIRGs and ULIRGs ob- served by GALEX (Howell et al. 2010), originally selected from the IRAS Revised Bright Galaxy Sample (Sanders et al. 2003). Note that the GALEX photometry for several of the sources in the Howell et al. (2010) are slightly revised in U et al. (2012) to account for highly irregular morphologies associated with galaxy interactions. The IR and UV apertures are matched and taken as total.

It is worth noting that all of the original blue compact galaxies used to derive the IRX $-\beta$ relation in Meurer et al. (1999) are included in the Gil de Paz et al. (2007) sample; most of them are significantly redder (higher $\beta$ value) when measured with $G A L E X$, consistent with other works that have since updated the nominal IRX $-\beta$ relation (Takeuchi et al. 2010, 2012).

\section{2. $0<z<3.5$ Dusty Galaxies}

We use a sample of $>4000$ Herschel-selected DSFGs in the COSMOS field (Scoville et al. 2007, 2013; Capak et al. 2007) as our $z>0$ sample, which have full UV-to-radio photometric coverage. Herschel (Pilbratt et al. 2010) coverage of COSMOS was carried out with the PACs (Poglitsch et al. 2010) and SPIRE (Griffin et al. 2010) instruments as part of the PEP (Lutz et al. 2011) and HerMES (Oliver et al. 2012) surveys. The DSFGs' characteristics and selection $(>3 \sigma$ in two or more of the five Herschel bands from 100-500 $\mu \mathrm{m}$ ) are described in detail in Lee et al. (2013), who present their empirically determined spectral energy distributions from the UV through the far-infrared. The method we use to estimate galaxies' deboosted far-infrared flux densities is described in Roseboom et al. (2010) with updates in Roseboom et al. (2012). The sample can largely be regarded as a luminosity-limited sample, as selection is unbiased with respect to far-infrared spectral energy distribution (SED) shape (i.e., dust temperature); deboosted flux densities are estimated to be complete above $S_{\text {deboosted }} \approx 10 \mathrm{mJy}$ at $160 \mu \mathrm{m}, 250 \mu \mathrm{m}, 350 \mu \mathrm{m}$, and $500 \mu \mathrm{m}$ and above $\approx 6 \mathrm{mJy}$ at $100 \mu \mathrm{m}$. We refer the reader to Lee et al. (2013) for details on sample selection. DSFGs' astrometry and multiwavelength counterparts are identified via a cross-identification method using $24 \mu \mathrm{m}$ and $1.4 \mathrm{GHz}$ position priors down to $S_{24}=80 \mu \mathrm{Jy}$ and $S_{1.4 \mathrm{GHz}}=65 \mu \mathrm{Jy}$ (Roseboom et al. 2010, 2012). This technique is predicated on the notion that high-redshift DSFGs should either be $24 \mu \mathrm{m}$ or $1.4 \mathrm{GHz}$ detected; this is an accurate assumption at $z \lesssim 2$ (with only a $3 \%$ failure rate; see Magdis et al. 2011), but the sample is largely incomplete at higher redshifts where $24 \mu \mathrm{m}$ and $1.4 \mathrm{GHz}$ surveys are relatively insensitive to detecting $z>2$ galaxies.

Of the original 4546 Herschel-identified galaxies with $24 \mu \mathrm{m}$ or $1.4 \mathrm{GHz}$ counterparts, only 4165 have reliable photometric redshifts (Ilbert et al. 2009): $\approx 92 \%$ of the total sample. The accuracy of the photometric redshifts in COSMOS is reported as $\sigma_{\Delta z /(1+z)}=0.012$. It should be noted that the DSFGs without reliable redshifts $d o$ exhibit different optical/near-IR photometry than those with reliable redshifts. Most are undetected in all but a few bands. Without redshifts, it is difficult to characterize this subset of the population with respect to IRX $-\beta$, beyond claiming that they likely have IRX $>3$.

Since emission from active galactic nuclei (AGNs) has the potential to affect the results of this study-by potentially skewing the rest-frame UV colors bluer and boosting the IR luminosities in the mid-IR regime-we must remove sources with X-ray detections, strong power laws in the Spitzer IRAC and MIPS bands, and sources with unexpectedly high radio-toFIR ratios. Of the 4218 Herschel-detected sources in COSMOS, only 5 are directly detected by XMM Newton (Brusa et al. 2010) and a further 95 are detected by Chandra (Civano 


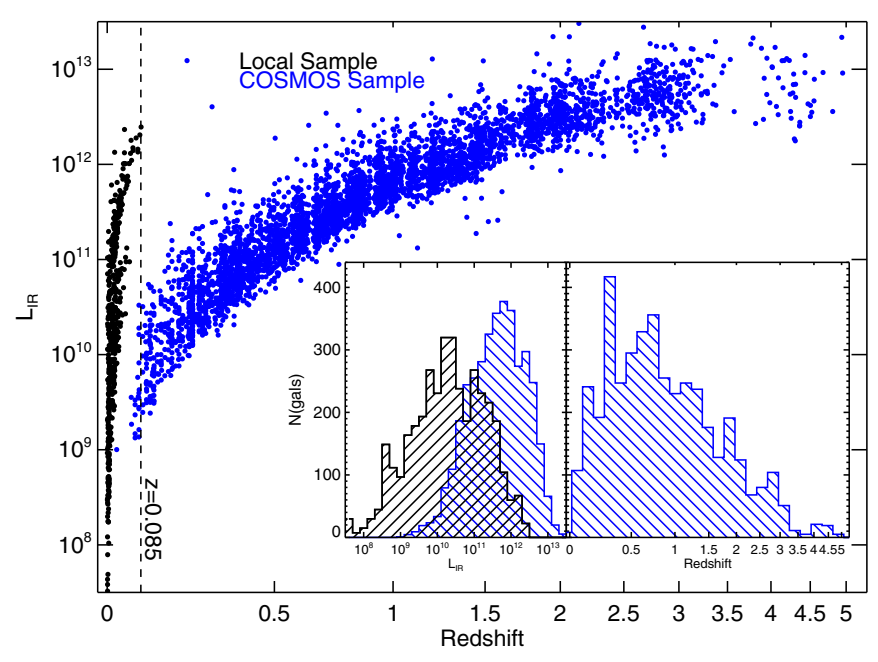

Figure 1. IR luminosity against redshift for both the local sample (black points) and the COSMOS $z>0$ sample (blue points). The inset shows histograms contrasting their IR luminosities (left) and redshifts (right). The local sample sits below $z=0.085$ while the median redshift for the COSMOS sample is 0.83 , with $60 \%$ of the sample sitting at $0.1<z<1$ and $98 \%$ below $z$ of 3.5 .

(A color version of this figure is available in the online journal.)

et al. 2012), suggesting powerful AGNs. They are removed from our analysis. While obscured AGNs are less likely to contaminate the rest-frame UV colors of their host galaxies, we also remove 332 additional galaxies suspected of hosting such AGNs, evidenced by a strong power law through the Spitzer IRAC and MIPS bands (Donley et al. 2012). All COSMOS DSFGs exhibiting very high radio-to-FIR ratios have been identified as AGNs through X-ray selection or mid-infrared power law. Of the remaining 4165 DSFGs in our study with photometric redshifts, a total of $432(\approx 10 \%)$ have been removed as potential AGN contaminants, leaving 3733 DSFGs for our analysis. Figure 1 plots the IR luminosities and redshifts of the COSMOS DSFG sample alongside the local sample for comparison. While $98 \%$ of the DSFG sample sits at $z<3.5$, the median redshift for the COSMOS sample is 0.83 , in close agreement with the measured peak redshift for Herschel-bright galaxies (Casey et al. 2012b).

\subsection{Calculations}

We fit $L_{\mathrm{IR}}$ consistently between the local and $z>0$ samples, from 8 to $1000 \mu \mathrm{m}$, and using all available infrared to millimeter data at rest-frame $8-2000 \mu \mathrm{m}$. For the local sample, this is primarily based on the four $I R A S$ bands at $12 \mu \mathrm{m}, 25 \mu \mathrm{m}, 60 \mu \mathrm{m}$, and $100 \mu \mathrm{m}$, with some additional data such as ScuBA (Dunne et al. 2003) for the brightest nearby (U)LIRGs. The full detailed photometry is described in U et al. (2012). The COSMOS sample includes the five-band Herschel 100-500 $\mu \mathrm{m}$ data, 24-70 $\mu \mathrm{m}$ Spitzer data, and, when available, AzTEC $1.1 \mathrm{~mm}$ data (Scott et al. 2008; Aretxaga et al. 2011) as well as ScuBA-2 $450 \mu \mathrm{m}$ and $850 \mu \mathrm{m}$ data (Casey et al. 2013; Roseboom et al. 2013). We use the far-infrared SED fitting technique described by Casey (2012); this has been shown to produce $L_{\mathrm{IR}}, T_{\text {dust }}$ and $M_{\text {dust }}$ estimates that are more accurate (when compared to direct interpolation of data) yet fully consistent with template fitting methods popular in the literature (e.g., Chary \& Elbaz 2001; Dale \& Helou 2002).

We compute the rest-frame UV luminosity of DSFGs by interpolating their measured photometry to rest-frame $1600 \AA$ as an AB apparent magnitude, $m_{1600}$, with

$$
L_{\mathrm{UV}}=\frac{4 \pi D_{\mathrm{L}}^{2} \nu_{1600}}{(1+z)} \times 10^{-\left(48.60+m_{1600}\right) / 2.5},
$$

where $D_{L}$ is the luminosity distance at redshift $z$ and $L_{1600}$ is given in $\mathrm{erg} \mathrm{s}^{-1}$. At $z \approx 0, \mathrm{UV}$ luminosity is the interpolation of FUV and NUV luminosity to $1600 \AA$ (which is approximately equal to $L_{\mathrm{FUV}}$ given the FUV filter's proximity to rest-frame $1600 \AA$ ). Note that as the redshifts of the galaxies in the sample increase, different observed bands are representative of the restframe UV: the NUV GALEX $0.231 \mu \mathrm{m}$ filter at $z \lesssim 0.6$, the Subaru $U$-band $0.346 \mu \mathrm{m}$ filter at $0.6 \lesssim z \lesssim 1.5$, and the Subaru $B$-band $0.460 \mu \mathrm{m}$ filter at $1.5 \lesssim z \lesssim 2$, and so on.

We then compute the rest-frame UV slope, $\beta$, using a powerlaw fit, where $\beta$ is defined by the relationship between flux and wavelength such that $F(\lambda) \propto \lambda^{\beta}$ (where flux is given in units of erg s $\mathrm{s}^{-1} \mathrm{~cm}^{-2} \AA^{-1}$ ). We calculate $\beta$ and the uncertainty on $\beta$ based on the multiple photometric measurements in the restframe UV regime available in COSMOS, namely, 1230-3200 A (Calzetti et al. 1994; Meurer et al. 1999; Calzetti 2001, this range is chosen to primarily consist of UV radiation from young $\mathrm{O}$ and $\mathrm{B}$ stars while also being wide enough to make the measurement of $\beta$ feasible using multiple photometric bands). Depending on redshift, $\beta$ is calculated with $2-5$ photometric bands, with an average of 3.3 bands per source. To determine if any systematic bias is introduced in the estimation of $\beta$ as a function of redshift (thus, as a function of the different broad and narrow bands available), we artificially redshift several different galaxy templates (from Bruzual \& Charlot 2003), convolve the templates with the available filter profiles, measure $\beta$, and compare to the intrinsic rest-frame UV slope. While we infer an intrinsic scatter in $\beta$ of up to 0.3 based on filter combination and noise, we infer no systematic variation.

Note that since the measurement of $\beta$ is done photometrically, it has the potential to be contaminated by stellar or interstellar absorption features and also enhanced extinction around the restframe $2175 \AA$ dust feature seen in high-metallicity environments like the Milky Way (but is less pronounced in low-metallicity environments like the LMC and SMC; Calzetti et al. 1994; Gordon et al. 2003). This $2175 \AA$ absorption feature is now known to exist in both low- $z$ galaxies (Conroy et al. 2010; Wild et al. 2011) and some high- $z$ galaxies (Buat et al. 2011, 2012; Kriek \& Conroy 2013); unfortunately, the intrinsic attenuation curve in highly dusty galaxies is more difficult to constrain since fewer rest-frame UV photons escape. We do not believe this substantially affects our results if the galaxies we study follow a Calzetti attenuation law, but we emphasize that further work-especially on the most extreme starbursts - is necessary to establish an empirical constraint on the relationship of attenuation to metallicity.

Another potential bias introduced by performing this calculation photometrically is possible contamination from emission lines, primarily Ly $\alpha$. If a photometric redshift is slightly lower than a galaxy's intrinsic redshift, emission from Ly $\alpha$ might boost the perceived rest-frame FUV emission, indicating a bluer UV slope. To test for this contamination, we compare the best-fit $\beta$ from the 1230-3200 $\AA$ range with a more restrictive 2000-3200 $\AA$ range, which should be substantially less affected by $\operatorname{Ly} \alpha$ contamination for galaxies where photometric redshifts are better than $\Delta z=0.5$. We find no systematic difference between the two different fits to $\beta$ and therefore argue that emission line contamination of $\beta$ in our sample is negligible and/or unlikely. 

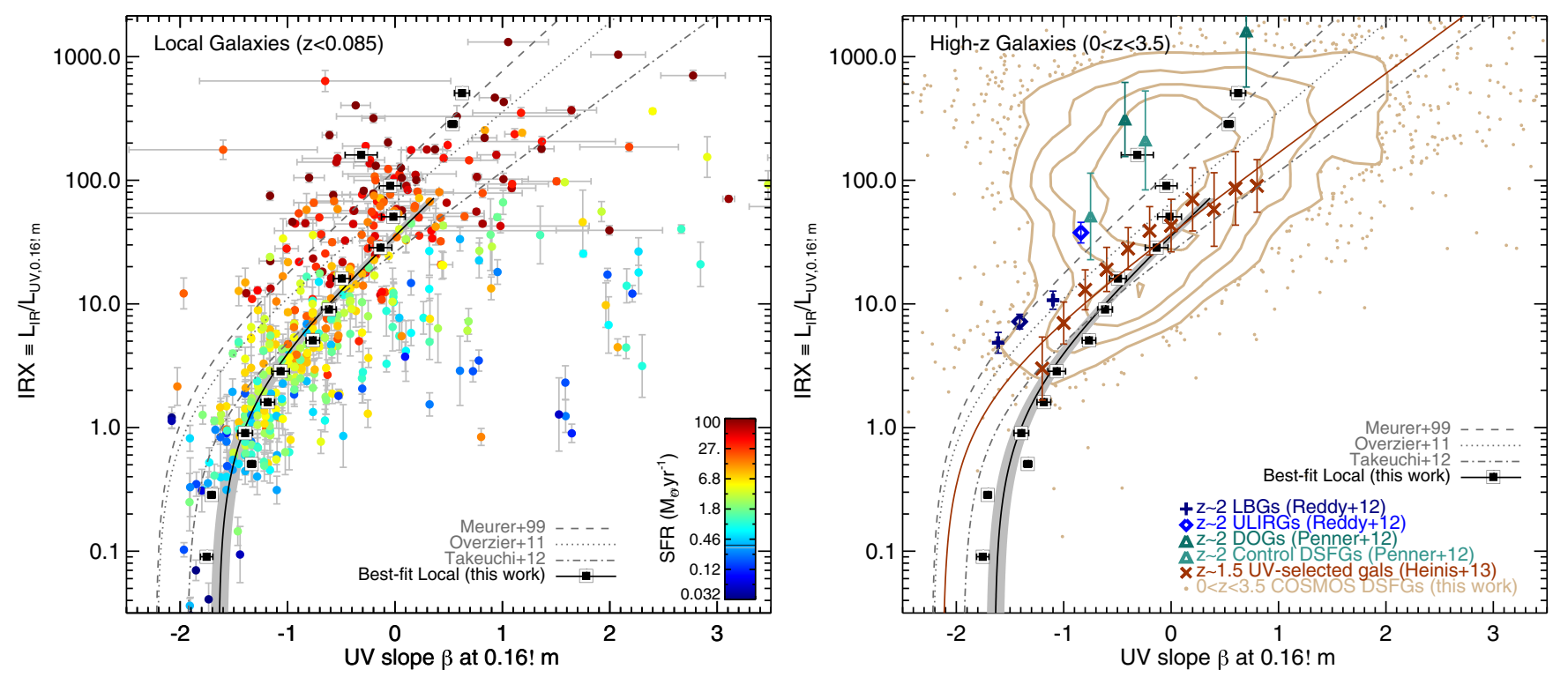

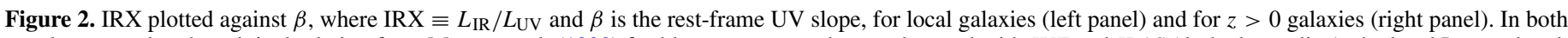

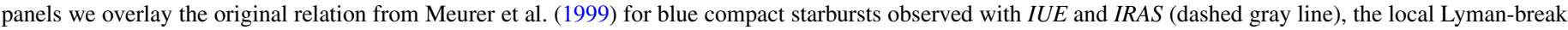

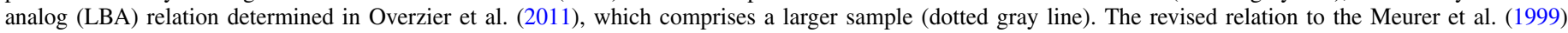

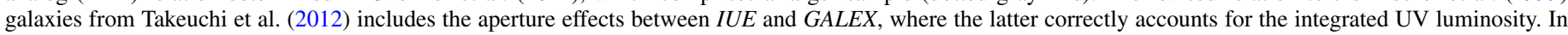

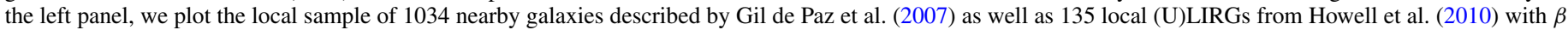

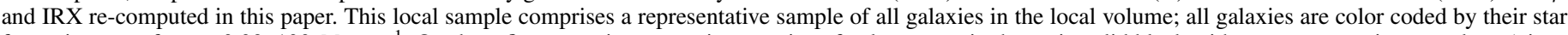

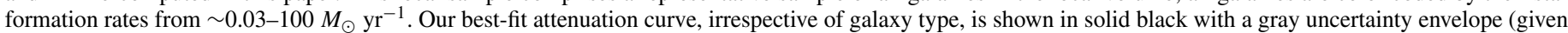

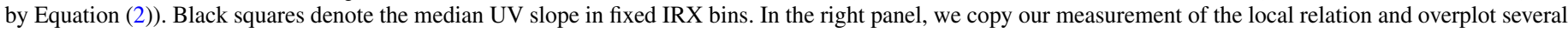

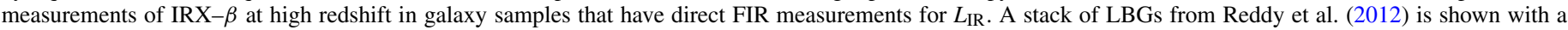

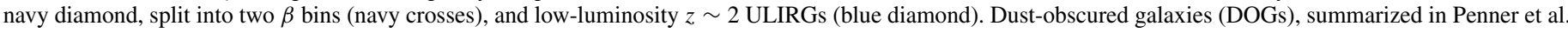

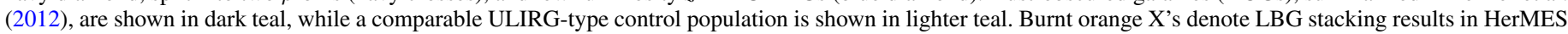

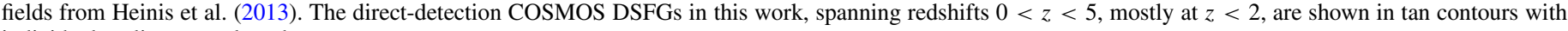
individual outliers overplotted.

(A color version of this figure is available in the online journal.)

We have corrected for Galactic extinction effects on both UV luminosity and $\beta$ using the dust maps of Schlegel et al. (1998), with updates provided by Peek \& Graves (2010); this is of particular importance for the nearby sample, which is distributed across the sky. We use the Milky Way attenuation curve $\left(A_{\lambda} / A_{V}\right.$ from Gordon et al. 2003) and the average extinction to reddening relation at the $V-$ band of $A_{V}=3.1 E(B-V)$ for the diffuse Milky Way (Cardelli et al. 1989) to determine appropriate correction factors $A_{\lambda}$ for observed bands from the FUV to the $z$ band.

\section{ANALYSIS}

\subsection{IRX- $\beta$ for Nearby Galaxies}

The left panel of Figure 2 shows the IRX- $\beta$ relation in the local Gil de Paz et al. (2007) and Howell et al. (2010) samples after re-measuring $L_{\mathrm{IR}}, L_{\mathrm{UV}}$, and $\beta$ uniformly, as described in Section 3.3. Galaxies' SFRs are denoted with color, ranging from blue to red, roughly increasing monotonically with IRX. It is clear that of the three calibrations of this relationship in the local universe, that of Takeuchi et al. (2012) provides the best fit, with both the widely used Meurer et al. (1999) and Overzier et al. (2011) fits offset in $\beta$ by $\sim 0.7$ toward the blue. Again, this disagreement arises from the corrected, integrated aperture of GALEX between the Meurer et al. (1999) and Takeuchi et al. (2012) works. While Overzier et al. (2011) do correct for aperture effects, they include a sample of particularly blue LBAs at low redshift which causes the median fit to be substantially bluer than that of Takeuchi et al. (2012). All three prior literature works are blueward of our fit at low IRX because of the subset of galaxies used to derive the fit: starbursts. Our inclusion of the Howell et al. (2010) LIRG data and the full Gil de Paz et al. (2007) heterogeneous sample spans a larger dynamic range in SFRs. If we exclude LIRGs and low SFR systems, we recover a shallower, bluer slope.

To determine the best-fit attenuation curve for local galaxies, we first bin up our data in IRX intervals of 0.25 dex. We choose to bin in IRX instead of $\beta$, as has been done elsewhere in the literature, to avoid degeneracies at $\beta<-1.5$ (where a wide range of IRX values all correspond to the same $\beta$ ) and at $\beta>0.5$ (where dusty galaxies become non-negligible with high values of IRX for the same $\beta$ ). We note that binning in $\beta$ or IRX produces consistent results between $-1.4<\beta<0$ and $1<\operatorname{IRX}<50$. Since these data are notably non-Gaussian in $\beta$ at a given IRX, we compute a representative "mode" $\beta$ value by measuring the peak of the $\beta$ histogram for the given IRX bin; the black squares on Figure 2 are these mode values. Errors on the mode are determined by bootstrapping. We exclude galaxies with IRX > 60 from the fit, where there is clear deviation, a topic we will return to in subsequent sections. A fit including the data at IRX $>60$ is very shallow and a poor solution to all data above IRX $>1$; we determined the cutoff of IRX $>60$ iteratively by excluding the highest IRX bins until the reduced $\chi^{2}$ was below 1.5. Note that the galaxies at very high $\beta$ and low IRX are too few to affect the average binning or fit. We determine that

$$
\mathrm{IRX}=1.68 \times\left[10^{0.4[(3.36 \pm 0.10)+(2.04 \pm 0.08) \beta]}-1\right]
$$

provides a good fit to the local data, with a characteristic spread in $\beta$ of 0.59 . This spread represents the standard deviation in 
the difference of measured $\beta$ values from the expected $\beta$ value (at a given IRX and given Equation (2)). Here the rest-frame UV extinction in magnitudes is represented by the quantity $\mathrm{A}_{\mathrm{UV}}=3.36+2.04 \beta$. The factor of 1.68 accounts for the bolometric correction of the original 40-120 $\mu \mathrm{m}$ FIR studies to total IR luminosities ( $L_{\mathrm{IR}}$ integrated from 8 to $1000 \mu \mathrm{m}$ ). One can verify that 1.68 is an appropriate choice of bolometric correction by generating SEDs with a variety of temperatures (e.g., by using the fitting method of Casey 2012) and comparing the integral luminosities between the two wavelength ranges. Regardless, the exact value of the bolometric correction only is relevant for readers wishing to compare our best-fit $A_{U V}$ to other works that use similar notation.

Very dusty systems with IRX $>60$ are unexpectedly blue. Beyond IRX $>60$, the mode value of UV color $\beta$ hits a wall at $\beta \approx-0.1$. We note that this result is slightly different from the analysis of the local sample presented in Overzier et al. (2011), who only measured galaxies with IRX $\gtrsim 100$ as slight outliers; the difference is primarily due to the redder colors we measure in the low-luminosity, low-IRX systems than any discrepancy at the high-IRX end. Also note that while bluer than expected from the IRX $-\beta$ relation, these very dusty galaxies are not as blue as some LBGs in the early universe. For the rest of our analysis, unless stated otherwise, whenever we refer to the "local" or "nominal" IRX $-\beta$ relation, we are explicitly referring to the relationship derived here, represented by Equation (2).

\subsection{Context of IRX- $\beta$ at High-z}

The right panel of Figure 2 places the local IRX $-\beta$ relationship into context at higher redshift. The local relations, including the best-fit relation in Equation (2), are overplotted with some comparative data sets from the literature, all of which involve direct far-infrared and direct rest-frame UV measurements (other works using indirect methods have been omitted). We include the 114 LBG stacking result from Reddy et al. (2012), whose measurement sits auspiciously along the Meurer et al. (1999) local attenuation curve, and their 12 galaxy, low-luminosity ULIRG comparison sample, which sits at slightly elevated IRX relative to Meurer et al. We also include the IR-stacking results on $38000+$ UV-selected $z \sim 1.5$ galaxies from Heinis et al. (2013). Note that the Heinis et al. data agree remarkably well with our locally derived IRX- $\beta$ relation and not the Reddy et al. stacking results. We attribute this disagreement to the fact that the Heinis et al. sample is not color-selected. One puzzling aspect of the Heinis et al. sample is the continuation of the relation to very red colors, $\beta \approx 1$. What is different in these galaxies at $z \sim 1.5$ that allows for this "extra" reddening? We believe sample selection and binning on $\beta$ rather than IRX (in this case out of necessity since the galaxies are not directly detected in the infrared). The Heinis et al. sample does not include galaxies explicitly selected by their dust emission.

As we found with our local sample, an exclusion of galaxies selected at IR wavelengths skews the IRX $-\beta$ relation toward redder colors (consistent with what was found by Takeuchi et al. 2012). As such, we do not attempt to derive a best-fit $z>0$ IRX $-\beta$ relation, since it is quite clear from Figure 2 (right panel) that most DSFGs do not follow a strict IRX- $\beta$ relationship. This would only be possible if we had a larger dynamic range in IRX, similar to what is available for the local sample.

It is quite clear that sample selection impacts the interpretation of IRX- $\beta$. In relation to the UV-selected samples, the aggregate IRX and $\beta$ values presented for dusty galaxies in Penner et al.
(2012) are notably offset in a similar manner as the local LIRGs and ULIRGs of Howell et al. (2010), emphasizing that dusty galaxies are bluer than the nominal IRX $-\beta$ relation would predict. Where do the COSMOS DSFGs lie in relation to these other $z>0$ measurements? The tan background contours in the right panel of Figure 2 represent the COSMOS DSFGs. They range from being directly on, or even below, the local relation to being above the relation by $\sim 2$ dex. In the next section, we investigate the difference between DSFGs that lie on the relation and those sitting substantially above.

DSFG characteristics in the IRX $-\beta$ plane show strong migration with infrared luminosity, ultraviolet luminosity, and redshift. The infrared luminosity and redshift migration goes hand in hand, whereby sources at the highest redshifts have the highest IR luminosities, based on our DSFG IR flux density based selection. Indeed, Figure 3 shows that contours of equal $L_{\mathrm{IR}}$ and $z$ roughly trace one another. On the other hand, contours of constant UV luminosity are orthogonal to those of constant $L_{\mathrm{IR}}$. This is expected given our IR sample selection and, importantly, assuming that there is only loose correlation between $L_{\mathrm{UV}}$ and $L_{\mathrm{IR}}$. From this plot, we can clearly see that galaxies with the highest IR luminosities and redshifts lie farthest away from the local attenuation curves in the IRX- $\beta$ plane. What does this tell us physically? To understand the underlying physical conditions leading to this $L_{\mathrm{IR}}$ - or $z$-driven migration of DSFGs in the IRX $-\beta$ plane, we first have to ensure that this is not caused by sample selection effects.

\subsection{Testing for Selection Bias}

The COSMOS field where the DSFGs are selected has the benefit of very deep ancillary data from the UV through the near-infrared for use in fitting stellar emission (Capak et al. 2007). To test for selection biases in the IRX $-\beta$ plane, we first complete an analysis of the depth of coverage in every restframe UV filter and rest-frame IR filter used to calculate $L_{U V}$, $\beta$, and $L_{\mathrm{IR}}$. In the rest-frame UV, we determined completeness as a function of magnitude for all galaxies in the field for each filter, using the photometry reported by Ilbert et al. (2010). In the far-infrared, we know the characteristic depth of Herschel coverage at $100 \mu \mathrm{m}, 160 \mu \mathrm{m}, 250 \mu \mathrm{m}, 350 \mu \mathrm{m}$, and $500 \mu \mathrm{m}$ as reported by Lutz et al. (2011) and Nguyen et al. (2010) and how to apply deboosting factors as appropriate to estimate intrinsic flux densities from raw, corrected for confusion noise (Roseboom et al. 2010, 2012). This single characteristic flux limit is primarily blurred by the uncertainty in deboosting factors (and to a much lesser extent, variations in instrument noise).

To tease out the possible selection biases in the IRX $-\beta$ plane, we construct a grid across $-3<\beta<3$ and $-2<\log ($ IRX) $<$ 4 , with a bin size of 0.05 in both quantities. Similarly, we construct a parallel grid of plausible IR luminosities and redshifts, which reaches far beyond the range of our observed sample: $6<\log \left(L_{\mathrm{IR}}\right)<14$, with a bin size of 0.05 and $0<z<5$ with $\Delta z=0.01$. For a given $\beta$ and IRX, at a given $L_{\mathrm{IR}}$ and $z$, we compute $L_{\mathrm{UV}}$ (where $L_{\mathrm{UV}}=L_{\mathrm{IR}} / \mathrm{IRX}$ ), rest-frame magnitude $m_{1600}$ at $1600 \AA$ via Equation (1), and assuming $F \propto \lambda^{\beta}$, we compute the observed $\mathrm{AB}$ magnitudes in the COSMOS filters that span the rest-frame UV at the given redshift $z$. We then add statistical noise (instrumental and confusion) to the measured magnitudes to be consistent with real data noise, remeasure $\beta$, and determine the probability that the given source would be detectable in our survey given the completeness curves described in the prior paragraph. This test is then repeated across the entire grid of possible $\beta$, IRX, 


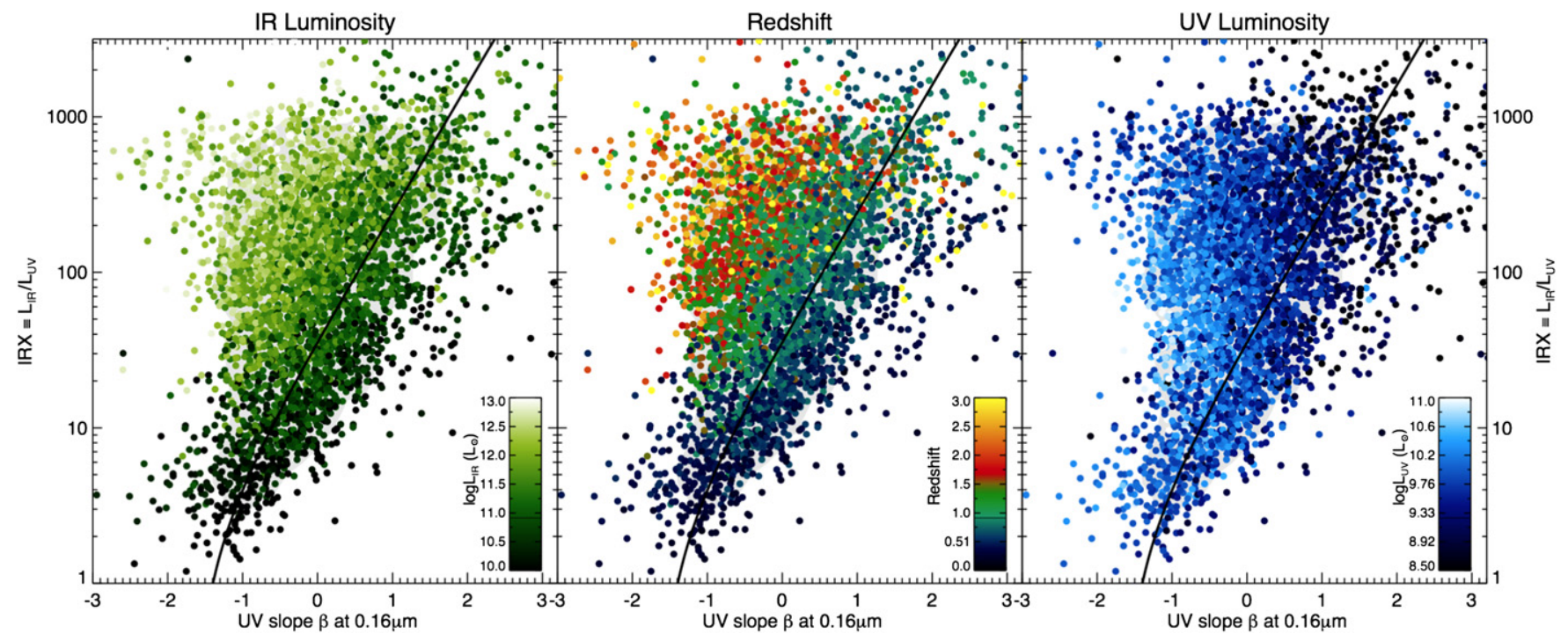

Figure 3. Three panels illustrating the IRX- $\beta$ relation with respect to DSFGs' IR luminosity (left), redshift (middle), and UV luminosity (right), as indicated by point color. The nominal IRX- $\beta$ relation as given in Equation (2) is shown in solid black in each panel for context. The higher a galaxy's IR luminosity or redshift, the farther away that galaxy sits from the IRX- $\beta$ relation toward bluer rest-frame UV colors, i.e., the steepest gradients in IR luminosity and redshift are orthogonal to the IRX $-\beta$ relation. On the other hand, the steepest gradient in UV luminosity is parallel to the IRX $-\beta$ relation.

(A color version of this figure is available in the online journal.)

$L_{\mathrm{IR}}$, and $z$ values, ${ }^{16}$ measuring a probability of inclusion in our DSFG sample for each permutation.

To complete this test of selection bias, we must also assign a probability of detection based on redshift and infrared luminosity (two of the four independent parameters in the above test for rest-frame UV detectability). This requires translating our Herschel flux density limits into luminosity, which requires some assumption on IR SED characteristics, primarily dust temperature. Symeonidis et al. (2013) and Lee et al. (2013) both present an observed trend of increasing dust temperature with luminosity that is unbiased with respect to temperature, unlike similarly luminous systems selected at $850 \mu \mathrm{m}$ (Blain et al. 2004; Chapman et al. 2004a; Casey et al. 2009). This relation is also observed locally, but shifted toward slightly warmer temperatures, perhaps indicative of size differences in the population, local galaxies being more compact (Swinbank et al. 2013). This dust temperature shift at high redshifts is explicitly illustrated in Figure 25 of Casey et al. (2014). We adopt a representative SED shape model to characterize the dust temperature, or SED peak wavelength, in terms of redshift and IR luminosity via

$$
\left\langle\log \lambda_{\text {peak }}\right\rangle=\eta\left[\log L_{\mathrm{IR}}-\left(\log L_{0}+\gamma \log (1+z)\right)\right]+\mu .
$$

At $z=0$, this equation simplifies to $\left\langle\log \lambda_{\text {peak }}\right\rangle=\eta\left(\log L_{\mathrm{IR}}-\right.$ $\left.\log L_{0}\right)+\mu$. Here $\eta=-0.062$ is the slope of the correlation, $\log L_{0}=10.60$ sets an arbitrary luminosity zero point at $z=0$, and $\mu=1.99$ is the average $\log \lambda_{\text {peak }}$ at that luminosity. The redshift evolution of $L_{0}$ is assumed to take the form $(1+z)^{\gamma}$, as is often used to model the evolution of $L_{*}$ in luminosity functions (e.g., Caputi et al. 2007; Goto et al. 2010), with best-fit $\gamma=2.7$. This function has characteristic scatter around $\log \lambda_{\text {peak }}$ of 0.045 (C. M. Casey et al. in preparation). This model gives us a reliable and realistic estimate for the limiting luminosity of our Herschel data. Note, however, that the temperature-redshift dependence of this model only weakly impacts our conclusions regarding

\footnotetext{
${ }^{16}$ As an aside, our tests indicate that there is no systematic bias in measuring
} $\beta$ at low luminosities. detectability in this paper, and only at the low IRX end, and is not a function of the rest-frame UV slope $\beta$.

Figure 4 offers an illustration of our survey's selection effects in the IRX $-\beta$ plane in two redshift slices. Areas shaded in tan are likely to be covered by the detection limits of COSMOS data. To answer the question of whether or not our deviation toward bluer colors is driven by selection effects, we model a population of $10^{6}$ galaxies that cluster about the local IRX- $\beta$ curve (from Equation (2)) with measured scatter. After applying our selection limits to that sample and binning in IRX, we are left with the rounded red points in Figure 4, which are significantly more red than the observed samples (shown in blue). This leads us to conclude that selection effects do not drive this observed blue $\beta$ offset in DSFGs.

\subsection{Comparison to Literature IR-selected Galaxies}

Our result that high-SFR, high- $L_{\mathrm{IR}}$ galaxies are much bluer than they would nominally be expected to be ${ }^{17}$ is consistent with previous analyses on smaller case studies of luminous infrared galaxies, both at low $z$ (Goldader et al. 2002; Howell et al. 2010) and high $z$. The offset from the IRX $-\beta$ relation was attributed in Goldader et al. (2002) to the significant spatial disassociation of the UV-luminous and IR-luminous portions of each galaxy. Goldader et al. speculated (at a time when very little was known about high- $z$ DSFGs) that local ULIRGs might be unusual in that their physical compactness makes it difficult for much restframe UV light to escape (i.e., at a given UV slope $\beta$, a local ULIRG will have a higher $L_{\mathrm{IR}} / L_{\mathrm{UV}}$ ratio than a normal star forming galaxy due to its compactness) and at high $z$, this might not be problematic if DSFGs are more spatially extended, as has often been found to be the case (e.g., Ivison et al. 1998, 2011; Hodge et al. 2012).

The SMG population (Smail et al. 1997), like the local ULIRGs, was found to be "bluer" than expected given the incredible $L_{\mathrm{IR}} / L_{\mathrm{UV}}$ ratios present, $\gtrsim 100$ (Smail et al. 2004). Chapman et al. (2005) pointed out that this implied that UV-based

\footnotetext{
17 Where the "expected" $\beta$ for a given IRX would be described by our Equation (2).
} 

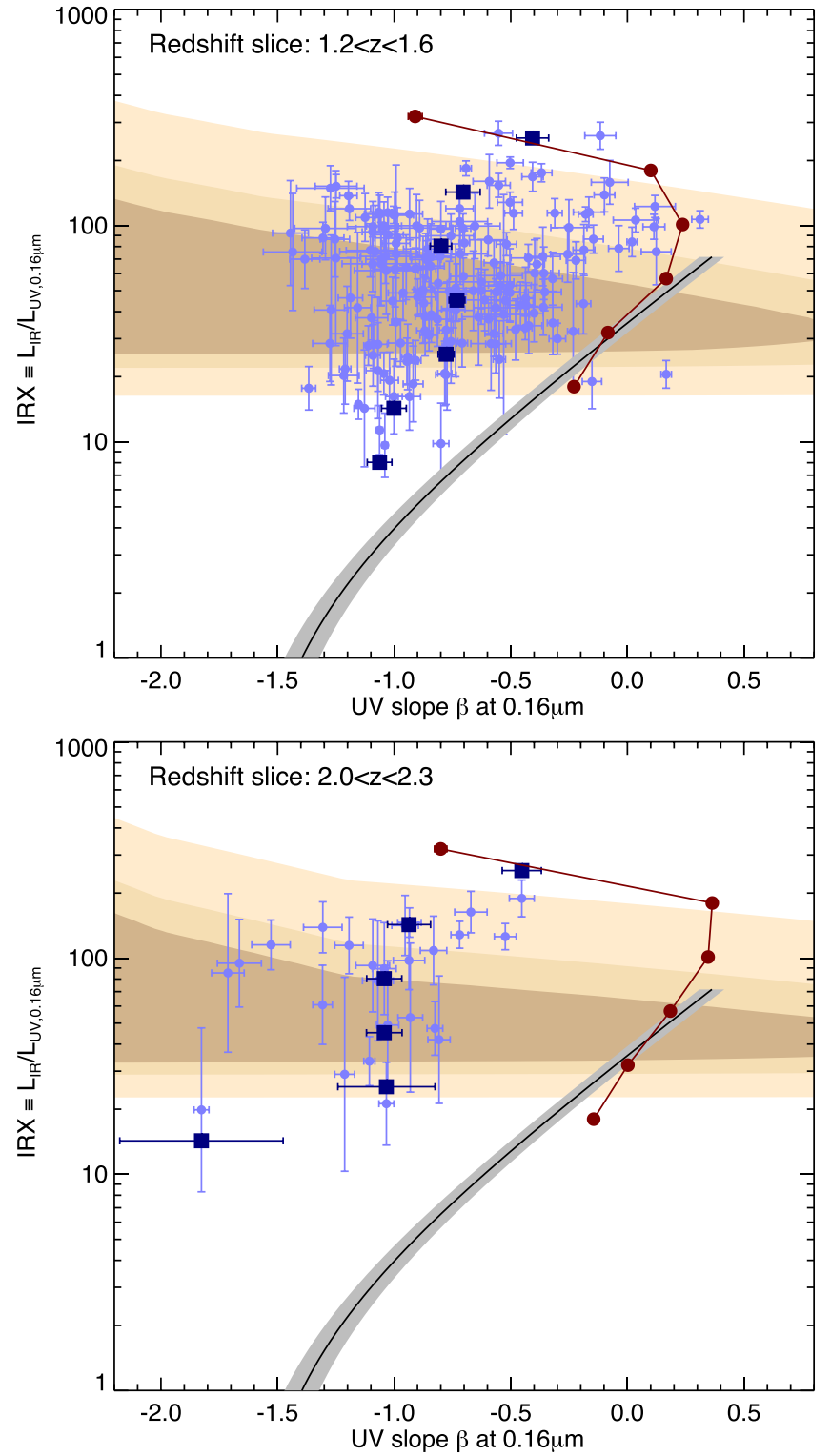

Figure 4. Illustration of the selection effects within our DSFG sample in two redshift slices. Light blue points are individual DSFGs that fall in the given redshift bins, and dark blue square points are the median values of $\beta$ for DSFGs falling in the given IRX bins. The tan shaded areas isolate accessible IRX- $\beta$ space where sources are over $95 \%, 90 \%$, and $80 \%$ likely to be included in our survey. The limit at low IRX is caused by the lower limit for direct IR detection combined with an upper limit on $L_{\mathrm{UV}}$. The limit at high IRX, which is a shallow function of $\beta$, depends on the depth of the UV/optical bands in COSMOS We overplot the local relation from Figure 2 in black (gray uncertainty) and simulate the selection functions impact on the local relation (round red points). This demonstrates that the offset toward lower $\beta$ in DSFGs is not driven by selection effects.

(A color version of this figure is available in the online journal.)

SFRs of SMGs underestimated their total SFRs by factors as large as $\sim 120$ even after the UV SFRs were corrected for extinction (see Adelberger \& Steidel 2000). Penner et al. (2012) also found that dusty galaxies - even when they are pre-selected to be incredibly red at optical wavelengths - have rest-frame UV characteristics bluer than expected given the local attenuation curve. Furthermore, a comparison control sample of IR-selected galaxies that are not DOGs revealed a similar, yet less extreme, result, completely in line with expectation given our results.
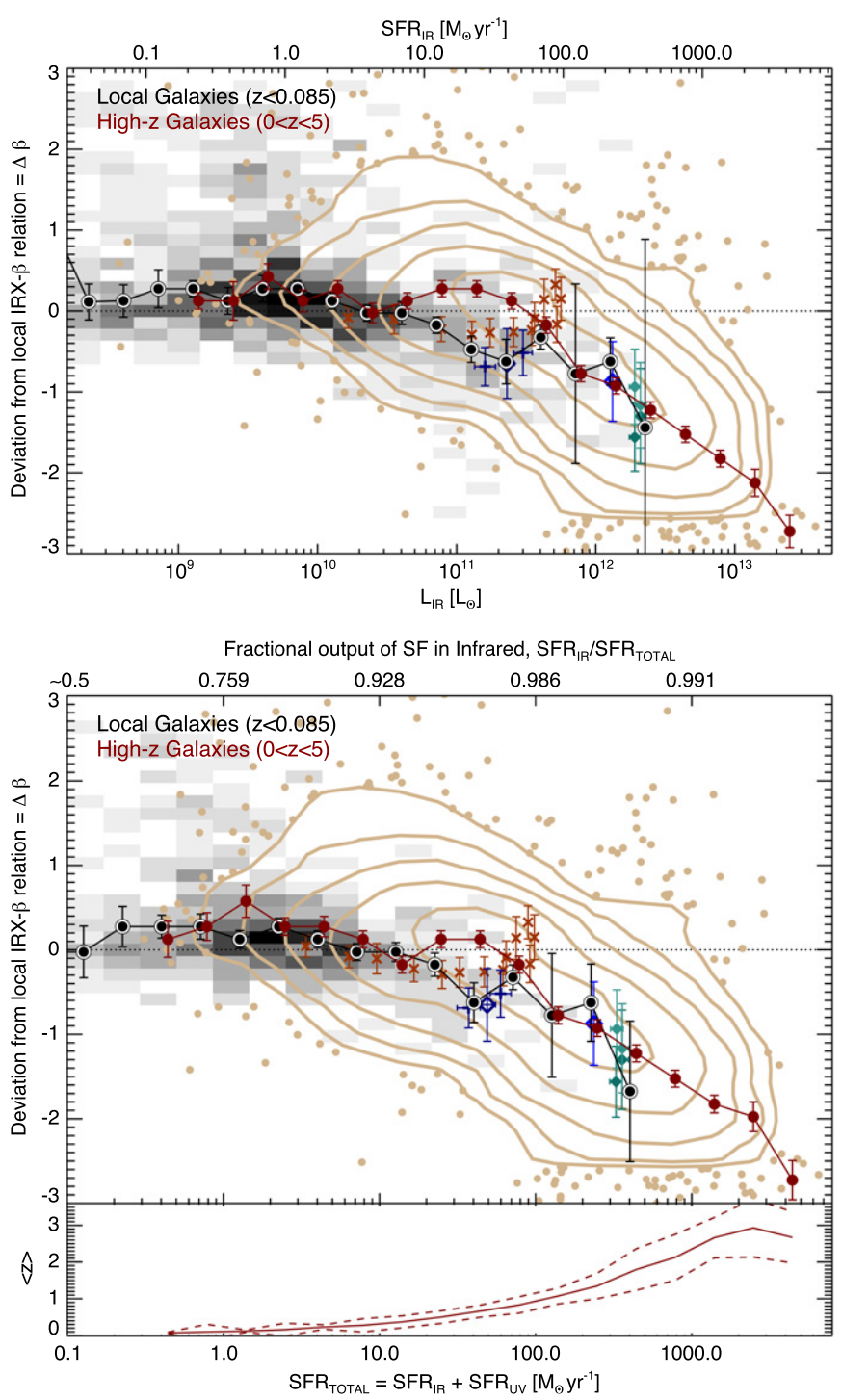

Figure 5. Deviation from the nominal IRX $-\beta$ relation, measured as a difference in rest-frame UV slope, $\Delta \beta \equiv \beta_{\mathrm{i}}-\beta_{\exp }$, where $\beta_{\exp }$ relates to IRX via Equation (2), against IR luminosity (top) and total star formation rate (bottom). Here we use $\mathrm{SFR}_{\mathrm{IR}}+\mathrm{SFR}_{\mathrm{UV}}$ as a proxy for total star formation rate, which, above $\gtrsim 10 M_{\odot} \mathrm{yr}^{-1}$, has $90 \%$ of its energy output in the IR (see the top axis of bottom plot). The local samples are shown as a shaded gray backdrop and overplotted black median values, while the COSMOS DSFGs are contoured in tan with median values in dark red. The median redshift of the sample at a given SFR is shown in the bottom inset ranging from $0<z<3$. Literature studies with published $L_{\mathrm{IR}}$ values are overplotted with the same symbols as in Figure 2 (right). In both diagrams, we see a strong break at $L_{\mathrm{IR}} \approx 10^{11.5} L_{\odot}$ or $\mathrm{SFR} \approx 40 M_{\odot} \mathrm{yr}^{-1}$, above which galaxies of all epochs are bluer than expected. (A color version of this figure is available in the online journal.)

\subsection{Deviation from the IRX- $\beta$ Relation}

From Figure 3, we gather that the deviation from the nominal IRX $-\beta$ relation is either a result of IR luminosity or redshift. Because local ULIRGs also deviate from IRX $-\beta$, we attribute the correlation of galaxies' deviation to their IR luminosities. Figure 5 investigates this deviation as a function of $L_{\mathrm{IR}}$. Due to the degeneracy of IRX at low $\beta$, we measure the deviation from the nominal IRX $-\beta$ relation (that given by Equation (2)) as the difference in UV slope, or $\Delta \beta$. Howell et al. (2010) present a similar plot in terms of $\Delta$ IRXfor the local sample (black/ gray shaded background on our plot). Although there is large scatter in $\Delta \beta$, owing to the complex star formation histories in 


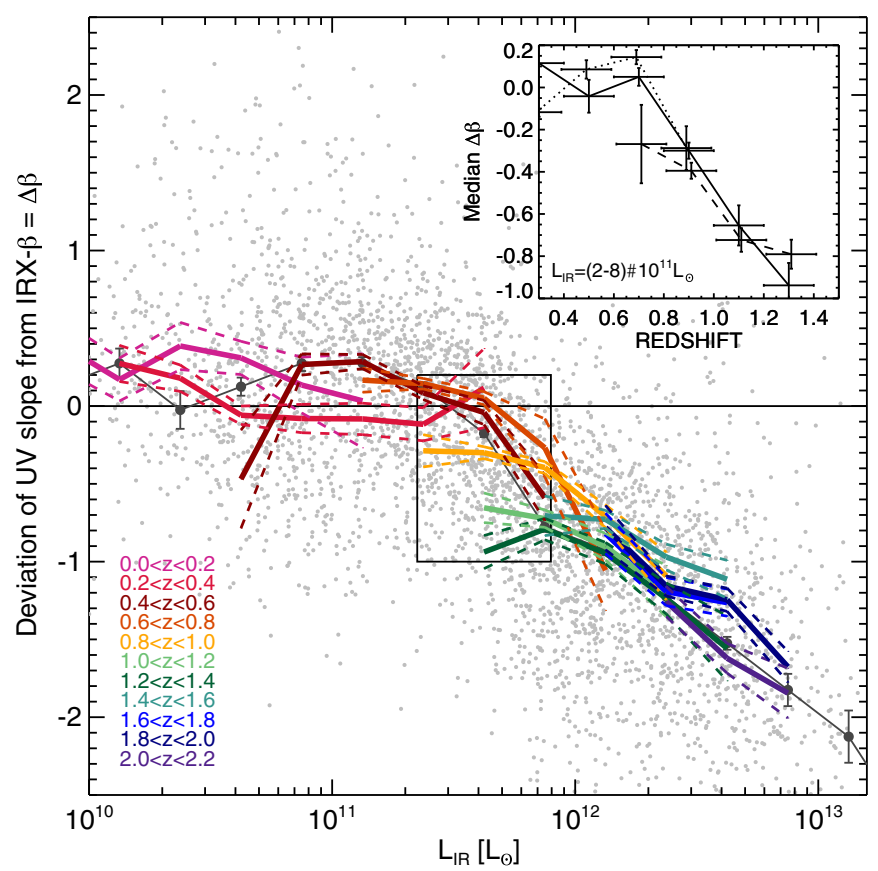

Figure 6. Same as Figure 5 , but split into $\Delta z=0.2$ redshift bins to probe a possible underlying redshift evolution distinct from the overall luminositydriven IRX $-\beta$ deviation. The weighted mean $\Delta \beta$ value for a given redshift bin and luminosity bin is shown with a bootstrap-estimated uncertainty. The only significant redshift evolution observed is seen between $0.6<z<1.4$ and $2-8 \times 10^{11} L_{\odot}$ (central box), whereby galaxies at lower redshift are redder by $\Delta \beta=1$. Galaxies at $z<0.6$ and $L_{\mathrm{IR}}<2 \times 10^{11} L_{\odot}$ are too few in number to measure evolution, while evolution is simply not seen in the higher luminosity bins above $z>1.4$. The inset plot shows the change in $\Delta \beta$ with redshift within this interval for the three luminosity bins at $2.4 \times 10^{11} L_{\odot}$ (dotted), $4.2 \times 10^{11} L_{\odot}$ (solid), and $7.5 \times 10^{11} L_{\odot}$ (dashed).

(A color version of this figure is available in the online journal.)

a heterogeneous galaxy population, both the local and $z>0$ samples share a characteristic "break" luminosity, above which galaxies systematically deviate from IRX $-\beta$ toward bluer UV slopes. The strength of the deviation increases with increasing luminosity. The "break" luminosity for the local sample appears to sit at $\approx 10^{11-11.5} L_{\odot}$ while the break in the COSMOS DSFG sample lies clearly at $\approx 10^{11.5} L_{\odot}$.

Following Kong et al. (2004) and some discussion presented in Reddy et al. (2006), this deviation at high $L_{\mathrm{IR}}$ is anticipated. Galaxies with more intense, more recent star formation will be intrinsically bluer for a fixed dust attenuation, because the underlying emission at UV wavelengths is dominated by a higher proportion of young $\mathrm{O}$ stars, contributing to the stellar continuum emission at 1216-1600 $\AA$. In the dusty star forming environments of ULIRGs where attenuation is substantial, $L_{\mathrm{IR}}$ can be directly mapped to the total SFR. The break in the $L_{\mathrm{IR}}-\Delta \beta$ plot correlates with increasing SFR. The bottom panel of Figure 5 investigates $\Delta \beta$ as a direct function of $\mathrm{SFR}_{\text {total }}$, or $\mathrm{SFR}_{\mathrm{IR}}+\mathrm{SFR}_{\mathrm{UV}}$. To emphasize the relative contributions of IR and UV to $\mathrm{SFR}_{\text {total }}$, the top axis of the bottom panel indicates the fractional output of star formation in the infrared. Similar to the break IR luminosity quoted above, the break in SFR is seen at $\approx 30-50 M_{\odot} \mathrm{yr}^{-1}$ in both local and $z>0$ samples (assuming a Salpeter IMF; Salpeter 1955).

Although the deviation from IRX $-\beta$ is clearly systematic above a given $L_{\mathrm{IR}}$, the huge scatter of $\sigma_{\Delta \beta}=1$ leads us to ask whether or not there is also any underlying redshift evolution. Figure 6 breaks up the $L_{\mathrm{IR}}-\Delta \beta$ plot into redshift bins with
$\Delta z=0.2$, where the tracks in redshift are representative of the weighted mean and uncertainties are bootstrapped. Slight differences between median $\Delta \beta$ exist with redshift and are seen most prominently in the half decade of $L_{\mathrm{IR}} \approx 10^{11.5-12} L_{\odot}$. The two luminosity bins at $4 \times 10^{11}$ and $7.5 \times 10^{11} L_{\odot}$ show substantial evolution between $z=0.6$ and $z=1.4$. Over the corresponding cosmic time, the median UV color in galaxies of equal luminosity shifts by $\Delta \beta=1$, i.e., it is substantially redder at lower redshifts. At higher luminosities (and also higher redshifts), no significant differences are detected between epochs.

Two plausible explanations for this observed "reddening" of matched- $L_{\mathrm{IR}}$ galaxies seen between $0.6<z<1.4$ are (1) an increasing metallicity of galaxies toward lower redshifts, or (2) different star formation histories present in galaxies at $z=1.4$ versus $z=0.6$. In the former case, it should be noted that some studies investigating the rest-frame UV continuum properties of optically selected galaxy populations show evidence for intrinsically bluer colors in lower metallicity systems (Alavi et al. 2014; Castellano et al. 2014), perhaps owing to a more top-heavy IMF in lower-metallicity galaxies (e.g., Marks et al. 2012). Note also that Marks et al. (2012) argue that a more topheavy IMF will manifest in environments with dense molecular clouds like starbursts, resulting in bluer rest-frame UV slopes.

The second explanation for the observed reddening with epoch is a different star formation history between LIRGs at $z=0.6$ and $z=1.4$. Following Kong et al. (2004), we note that galaxies with more recent burst histories and younger stellar populations are expected to have bluer rest-frame UV slopes. This suggests, in fact, that our $z=1.4$ DSFGs are likely younger and burstier than their $z=0.6$ analogues, a notion that might seem contradictory to recent works suggesting that LIRGs and ULIRGs at high $z$ are less likely to be burst-driven than their local counterparts, based on the galaxy main-sequence (e.g., Noeske et al. 2007; Elbaz et al. 2011; Rodighiero et al. 2011; Nordon et al. 2012).

Besides redshift evolution, the large scatter in color in each luminosity bin could also be partly due to differences in the intrinsic dust attenuation curves, where flatter curves indicate larger attenuations (IRX) for the same color ( $\beta$ Gordon et al. 2000; Burgarella et al. 2005), or viewing angle effects. Studying the morphological characteristics of the bluest and reddest sources of a given luminosity or SFR to probe inclination, bulgeto-disk ratio, and interactions will be a necessary and worthy follow-up study, and yet is beyond the scope of this paper.

It is worth highlighting that although we offer many plausible physical explanations here to describe the deviation (or possible blueward $z$ evolution), we primarily attribute the bulk shift off IRX $-\beta$ to dust geometry effects. As we will later discuss in more detail in Section 6, we know that nearby LIRGs and ULIRGs are primarily enshrouded in a thick cocoon of dust, where the IR emission is high and the UV photons are few. The UV light that does escape does so in a patchy pattern, leaking out in bright concentrations. These small openings dominate the UV luminosity, therefore, the total UV color of the galaxy, while the dust enshrowded component (which is spatially disassociated) dominates $L_{\mathrm{IR}}$. We return to this in our discussion.

Note that the various other measurements of the IRX $-\beta$ relation at $z>0$ from LBGs and DOGs align with the observed deviation we measure for DSFGs at their respective IR luminosities, within uncertainty. Could this explain some of the discrepancies between, e.g., the Reddy et al. (2012) result-LBGs that lie blueward of our nominal IRX- $\beta$ fit - the Heinis et al. (2013) 
Table 1

DSFG Contaminants to High- $z$ LBG Searches

\begin{tabular}{|c|c|c|c|c|c|c|c|}
\hline \multirow{2}{*}{$\begin{array}{l}\text { Target } \\
\text { Redshift }\end{array}$} & \multirow[t]{2}{*}{ Selection Criteria } & \multicolumn{5}{|c|}{ DSFG CONTAMINANT CHARACTERISTICS } & \multirow{2}{*}{$\begin{array}{c}\% \\
\text { LBG } \\
\text { CONTAM. }\end{array}$} \\
\hline & & $N_{\text {contam }}$ & $\begin{array}{c}\rho_{\text {contam }} \\
\left(\operatorname{arcmin}^{-2}\right)\end{array}$ & $\begin{array}{l}\text { Av. App. } \\
\text { mag }\end{array}$ & $z_{\text {phot }}$ & Av. Implied & \\
\hline \multicolumn{8}{|c|}{ Optical-ONly LBG Dropout Selection } \\
\hline$z \sim 2.5$ & $\begin{aligned} {\left[U_{300}-B_{450}>1.1\right] \wedge\left[B_{450}-I_{814}\right.} & <1.5] \wedge \\
{\left[U_{300}-B_{450}>0.66\left(B_{450}-I_{814}\right)+1.1\right] } & \wedge[U>27]\end{aligned}$ & 15 & $2.4 \times 10^{-3}$ & (B) 26.7 & 2.7 & $-0.37 \pm 0.13$ & $0.01 \%$ \\
\hline$z \sim 4$ & $\begin{array}{c}{\left[B_{435}-V_{606}>1.1\right] \wedge\left[V_{606}-z_{850}<1.6\right] \wedge} \\
{\left[B_{435}-V_{606}>\left(V_{606}-z_{850}\right)+1.1\right] \wedge[U B>27]}\end{array}$ & 35 & $5.5 \times 10^{-3}$ & $(V) 26.3$ & 2.9 & $-0.30 \pm 0.18$ & $0.37 \%$ \\
\hline$z \sim 5$ & $\begin{aligned} & {\left[V_{606}-i_{775}>1.2\right] \wedge\left[i_{775}-z_{850}<0.6\right] \wedge } \\
& {\left[\left(V_{606}-i_{775}>0.9\left(i_{775}-z_{850}\right)\right) \vee\left(V_{606}-i_{775}>2\right)\right] } \\
& \wedge(U B V>27)\end{aligned}$ & 26 & $4.1 \times 10^{-3}$ & (i) 26.2 & 2.0 & $0.0 \pm 0.2$ & $3.2 \%$ \\
\hline$z \sim 6$ & 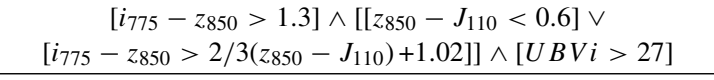 & 7 & $1.4 \times 10^{-3}$ & (z)26.1 & 2.0 & $-0.4 \pm 0.2$ & $8.7 \%$ \\
\hline \multicolumn{8}{|c|}{ Optical and Near-IR LBG Dropout Selection } \\
\hline$z \sim 4$ & $\begin{array}{c}{\left[B_{435}-V_{606}>1\right] \wedge\left[i_{775}-J_{125}<1\right] \wedge} \\
{\left[B_{435}-V_{606}>1.6\left(i_{775}-J_{125}\right)+1\right] \wedge[U B>27]}\end{array}$ & 6 & $9.4 \times 10^{-4}$ & $(V) 26.3$ & 2.9 & $-0.30 \pm 0.18$ & $<0.01 \%$ \\
\hline$z \sim 5$ & $\begin{array}{c}{\left[V_{606}-i_{775}>1.2\right] \wedge\left[z_{850}-H_{160}<1.3\right] \wedge} \\
{\left[V_{606}-i_{775}>0.8\left(z_{850}-H_{160}\right)+1.2\right]} \\
\wedge(U B V>27)\end{array}$ & 2 & $3.2 \times 10^{-4}$ & (i) 26.2 & 2.0 & $0.0 \pm 0.2$ & $0.01 \%$ \\
\hline$z \sim 6$ & $\begin{aligned} {\left[i_{775}-z_{850}>1.0\right] } & \wedge\left[Y_{105}-H_{160}<0.45\right] \wedge \\
{\left.\left[i_{775}-z_{850}>0.777\left(Y_{105}-H_{160}\right)+1.0\right]\right] } & \wedge[U B V i>27]\end{aligned}$ & 0 & $<1.6 \times 10^{-4}$ & (z)26.1 & 2.0 & $-0.4 \pm 0.2$ & $<0.04 \%$ \\
\hline$z \sim 7$ & $\begin{array}{c}{\left[z_{850}-Y_{105}>0.7\right] \wedge\left[J_{125}-H_{160}<0.45\right] \wedge} \\
{\left[z_{850}-Y_{105}>0.8\left(J_{125}-H_{160}\right)+0.7\right] \wedge[U B V i z>27]}\end{array}$ & 3 & $4.7 \times 10^{-4}$ & $(Y) 24.9$ & 1.9 & $-1.0 \pm 0.2$ & $0.40 \%$ \\
\hline$z \sim 8$ & $\begin{array}{c}{\left[Y_{105}-J_{125}>0.45\right] \wedge\left[J_{125}-H_{160}<0.5\right] \wedge} \\
{\left[Y_{105}-J_{125}>0.75\left(J_{125}-H_{160}\right)+0.525\right] \wedge[U B \text { BizY }>27]}\end{array}$ & 1 & $1.6 \times 10^{-4}$ & $(J) 24.1$ & 4.3 & $-1.11 \pm 0.14$ & $0.34 \%$ \\
\hline$z \sim 10$ & $\begin{array}{c}{\left[J_{125}-H_{160}>1.2\right] \wedge\left[H_{160}-[3.6]<1.4 \vee\right.} \\
S / N([3.6])<2] \wedge[U B V i z Y J>27]\end{array}$ & 0 & $3.2 \times 10^{-4}$ & $(H) 23.0$ & 2.8 & $-0.2 \pm 0.4$ & $<2.5 \%$ \\
\hline
\end{tabular}

Notes. The above selection criteria are outlined explicitly in Bouwens et al. (2009) for optical-only selection and Bouwens et al. (2014) for optical and near-IR selection, yet the selection method is very similar to other high- $z$ dropout selection techniques outlined in Bunker et al. (2003), Giavalisco et al. (2004), Beckwith et al. (2006), Stanway et al. (2007), Oesch et al. (2010), and Bouwens et al. (2011b). Magnitudes are interpolated from the observed COSMOS broad-, intermediate-, and narrow-band imaging to the given selection filters and the effective area of this search is performed in the $1.76 \mathrm{deg}^{2}$ area of UltraVISTA in $\operatorname{COSMOS~(~} \gtrsim 6$ times the area used in the deep dropout searches). In addition to the stated color selection criteria, we use a S/N cutoff in $U B V i z Y J$ bands as appropriate. The characteristics of the DSFGs satisfying the selection criteria are given: their average apparent magnitudes, implied $\beta$ if at the target redshift, their median photometric redshift (from the UltraVISTA near-IR-selected catalog; McCracken et al. 2012), and their density on the sky. The last column gives the percentage of LBG candidates, selected at the given target redshift, that are, in fact, DSFGs at lower redshifts. We use the stated number of candidates identified per square arcminute as stated in Bouwens et al. (2009) and Bouwens et al. (2014).

results-UV-selected galaxies lying very close to our nominal IRX- $\beta$ fit-and the Penner et al. (2012) results-where dustselected galaxies are notably bluer than our nominal IRX $-\beta$ fit? We think yes, that it may be understood as a function of these' galaxies total SFRs or IR luminosities. So while earlier we thought it auspicious that the $z \sim 2$ Reddy et al. LBGs aligned perfectly with the Meurer et al. (1999) IRX- $\beta$ relation (a relation with known biases and measurement discrepancies), here we can attribute that alignment to two different biases which together are skewed blueward of the nominal IRX $-\beta$ relation for a heterogeneous, normal galaxy population. It is also not surprising to see the much bluer colors in the Penner et al. (2012) DOGs given their high IR luminosities.

\section{HOW DO DSFGS IMPACT HIGH- $z$ GALAXY SEARCHES?}

The search for the highest-redshift galaxies, at $z>4$, is predicated on the Lyman break dropout technique (Steidel et al. 1996). DSFGs are usually thought to be too intrinsically red and too rare to impact high- $z$ LBG searches, but our results hint that a subset of DSFGs might satisfy LBG selection criteria, either because they are bluer than anticipated or, alterna- tively, their rest-frame optical emission lines might contribute substantially to broad-band photometry and be mistaken for a Lyman break at higher redshift (see Bouwens et al. 2011a). Although the LBG dropout selection technique has some advantages, e.g., providing an easily repeatable selection, it is potentially prone to more contamination than high- $z$ photometric redshift techniques, which are less biased against LBGs with intrinsically redder colors (Finkelstein et al. 2010, 2012). This section makes use of the extensive deep optical and near-IR data in the COSMOS field to explore the extent to which directly detected Herschel DSFGs might contaminate high-z LBG "dropout" searches.

LBG "dropout" selection at high $z$ is usually defined in terms of Hubble Space Telescope (HST) broadband filters. We use the $30+$ band photometry in the COSMOS field (most recently complete with deep near-IR imaging from UltraVISTA covering $1.76 \mathrm{deg}^{2}$; McCracken et al. 2012) to interpolate and predict magnitudes in these $H S T$ filters and then apply the various LBG selection criteria to our DSFGs, as outlined in Table 1. We use the LBG dropout selections that are outlined in Bouwens et al. (2009), for optical data only, and Bouwens et al. (2014), for optical and near-infrared data. In addition to the color cuts, we apply a magnitude cutoff of $27[\mathrm{AB}]$ in bands shortward 


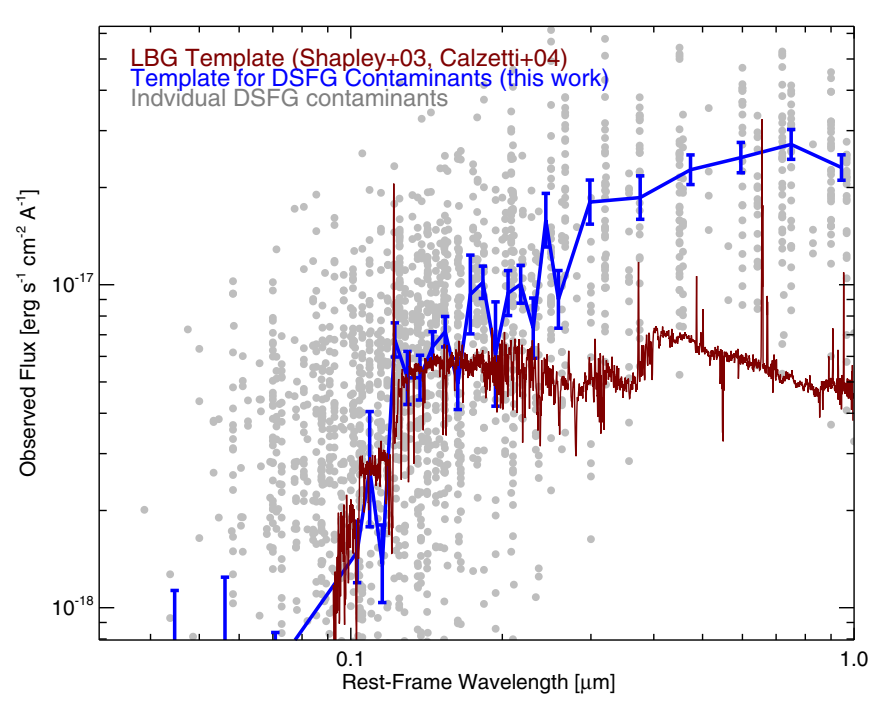

Figure 7. Average template SED for the DSFG population that contaminates LBG dropout searches, given in rest-frame wavelength at the intended target redshift. In gray, we show the individual photometric points for DSFG contaminants that satisfy each of the different redshift LBG cuts given in Table 1. The blue curve gives the average contaminant SED for the entire DSFG contaminating population, averaged over all selection redshifts (in most cases, the DSFG redshifts are dissimilar to the target redshifts). This contaminant template illustrates the need for rest-frame optical constraints on LBG SEDs that are anticipated to be slightly bluer than most DSFG contaminants. Note that very deep coverage shortward of the Lyman break does not guarantee highredshift identification, since most DSFGs also drop out of the bluest optical filters. The DSFGs' photometry is observed and no normalization to a flux scale is done.

(A color version of this figure is available in the online journal.)

of the Ly $\alpha$ break. This prevents against obvious lower-redshift bright contaminants and is preferred to a strict signal-to-noise ratio $(\mathrm{S} / \mathrm{N})$ cutoff since the COSMOS data are of varying depths compared to the deep HST imaging used to identify $z \gtrsim 4$ LBGs. The contaminating fraction of DSFGs in LBG searches is then computed by comparing their surface densities to those of LBG candidates of comparable magnitudes (as presented in Bouwens et al. 2009, 2014).

Table 1 summarizes our results. We find that at low redshifts, $z \lesssim 4$, the contamination of LBG searches with DSFGs is negligible $(\ll 1 \%)$, since LBGs at that epoch are extremely common relative to DSFGs. The DSFGs that do satisfy the LBG color cuts at these epochs do seem to sit at the appropriate redshifts and are the bluest of DSFGs, but again are negligible in number when compared to the much more common LBG. Without near-infrared selection criteria, dusty galaxies can easily pass LBG selection at $z \sim 5-6$ and comprise a significant fraction of contaminants, as much as $\approx 9 \%$ at $z \sim 6$. However, due to the recent deep surveys from the Wide Field Camera 3 in the near-infrared, most of those contaminants are thrown out, with rates as low as $0.1 \%$.

Figure 7 shows an average template for DSFGs that contaminate these high- $z$ LBG dropout searches. To construct the template, we first build a median template for contaminants in each LBG selection, corresponding to different redshifts, and then shift them into the same rest-frame wavelength grid: that which corresponds to the assumed redshift of selected LBGs. The median template for all of the LBG selection is then shown in blue, ${ }^{18}$ clearly showing the corresponding break that is mis-

\footnotetext{
18 Available for download now at

http://herschel.uci.edu/cmcasey/research.html under "Tools" and in the future available on the PI's research Web site under the same header.
}

taken for the Lyman- $\alpha$ break. Note that this template is not meant to be physical as it is a coaddition of DSFGs' observed SEDs at a variety of redshifts and, as such, the break observed is caused by a number of different physical processes, e.g., bright [O II] emission, bright $\mathrm{H} \alpha$, or a $4000 \AA$ or Balmer break. One primary difference seen between the expected SED of an LBG (see the composite in Figure 7; Shapley et al. 2003; Calzetti et al. 1994) and our contaminant template is that the rest-frame optical emission of LBGs is bluer than in DSFG contaminants.

Although this method of checking for dusty "contaminants" in UV galaxy searches has caveats, most notably mismatched photometric depths of the surveys and the likely incompleteness of DSFG samples beyond $z>2$, it serves as a worthy reality check for high- $z$ galaxy search campaigns. The increasing rate of contamination in the highest-redshift bins is due to both the degeneracies of colors over a relatively short rest-frame wavelength span and the diminishing surface density of higher- $z$ LBGs. Overall, contamination rates of $<2 \%-3 \%$ from dusty starbursts bode well for high- $z$ searches.

Besides providing an essential measurement of the universe's SFRD at very early times, high- $z$ LBG searches have led us to infer the dust content of the earliest galaxies using the IRX- $\beta$ relation (Bouwens et al. 2009). Because the distribution of rest-frame UV slopes of LBGs is bluer at earlier times, Bouwens et al. argued that these high- $z$ LBGs contain very little dust. Despite the degeneracy between blue dustless and dusty galaxies, our results-low contamination rates from DSFGs in LBG searches-corroborate the Bouwens et al. conclusions, that the majority of high- $z$ LBGs contain relatively little dust, by demonstrating that very few high-z LBGs will be directly detected with Herschel or similar submillimeter instruments. Of course, deep submillimeter follow-up (e.g., from ALMA) is needed to infer the dust content in individual high- $z$ LBGs (e.g., Chapman \& Casey 2009), in particular to constrain their SFRs and contribution to the total SFRD. This is because our results have only ruled out the tip of the iceberg: it is quite possible that blue LBGs in the early universe are much dustier than their UV slopes may infer yet still too faint to be detected in the existing Herschel data.

Furthermore, the existence of extreme, dusty galaxies at the same epochs should raise concern for our understanding of the integrated SFRD (e.g., Hopkins \& Beacom 2006) at these early times. The SFR in one DSFG can often exceed the SFR in individual LBGs by factors of 50-100. While here we have determined that a very low fraction of LBGs will be directly detected by Herschel, we note that (1) Herschel is far less sensitive to detecting $z>2$ starbursts than longer-wavelength submillimeter surveys (at $\approx 1 \mathrm{~mm}$; see Figure 7 of Casey et al. 2014), and (2) very few $z \sim 4-6$ DSFGs will be selected as LBGs. Of the 39 COSMOS DSFGs with photometric redshifts above 4 , only $20 \%$ satisfy LBG selection criteria. Furthermore, we know that high- $z$ extreme DSFGs like HFLS3 at $z=6.34$ do exist (Riechers et al. 2013; Cooray et al. 2014), yet the difficulty in obtaining spectroscopic identification has made the assessment of their volume density quite challenging (Dowell et al. 2014). While our comparison to LBGs illustrates that there is little concern for significant contamination, we emphasize that the $z>4$ SFRD is still highly uncertain without concrete constraints from direct far-infrared measurements.

\section{DISCUSSION}

Our results indicate that galaxies with particularly high SFRs, most of which are measured from their output in the far-infrared, 


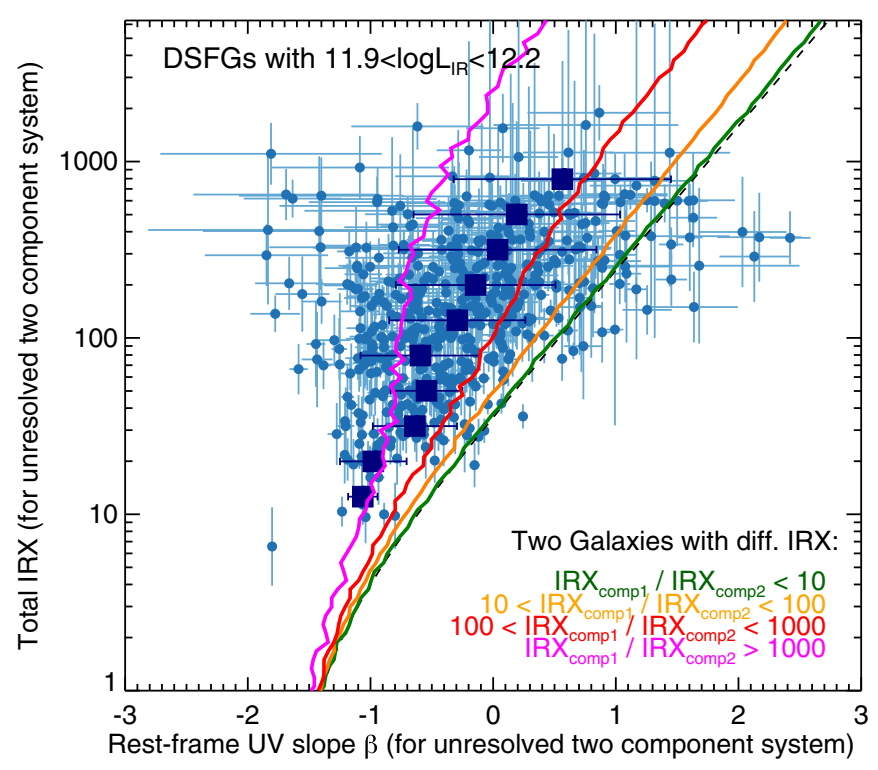

Figure 8. Can spatially disassociated UV- and IR-bright components explain the bluer rest-frame UV colors of DSFGs? Here we explore how two nearby galaxy components of different obscurations (IRX values) might be perceived on the IRX- $\beta$ plane if the two components are not resolved from one another. We simulate $10^{6}$ hypothetical pairs where all individual components lie on the IRX $-\beta$ relation (dashed black line) with observed local scatter \pm 0.59 in $\beta$. Only pairs with significant differences between component IRX values will be measured as having colors bluer than expected due to spatial disassociation. This simplified simulation is effectively modeling the relative bimodality of a galaxy's dust obscuration. We overplot all DSFGs within the narrow $L_{\mathrm{IR}}$ ranging corresponding to our simulation in light blue (median values are dark blue squares) for comparison. This figure suggests that most $10^{12} L_{\odot}$ DSFGs are bimodal, or have spatially disassociated components that differ in IRX by factors of $\sim 300$.

(A color version of this figure is available in the online journal.)

are bluer than expected given the nominal dust attenuation curve for low-luminosity, "normal" star forming galaxies. What underlying physical processes could be responsible for these bluer UV colors?

The first possible explanation is that the rest-frame UV emission and far-infrared emission are physically disassociated or spatially distinct (Goldader et al. 2002; Chapman et al. 2004b). Assuming that the systems are still physically bound, this might be due to a recent catastrophic event, like a merger-driven burst. Can geometric effects alone explain the systematically bluer rest-frame UV slopes in DSFGs? If a system consists of two dominant components, one with $L_{\mathrm{UV}} \approx L_{\mathrm{IR}}$ (unobscured) and one with $L_{\mathrm{IR}} \gg L_{\mathrm{UV}}$ (obscured), both of which follow the IRX $-\beta$ relation, the IRX value assumed for the entire system will be taken from the obscured component, since there the IR luminosity dominates, and the $\beta$ value from the unobscured component, which dominates all UV light; the result is an unresolved, blue DSFG with high IRX. However, take another system with its IR luminosity and UV luminosity well distributed in, e.g., a disk (for a simple test, if you were to split it into two components, as before, both would have roughly evenly matched $L_{\mathrm{IR}}$ and $L_{\mathrm{UV}}$ ). If the individual regions of this homogenized system all followed IRX- $\beta$, then the total would as well. Only dramatic spatial variations in the distribution of $L_{\mathrm{UV}}$ and $L_{\mathrm{IR}}$ can lift a system substantially above IRX- $\beta$.

Figure 8 illustrates how the relative dustiness (IRX values) between the two hypothetical, nearby components impacts how "blue" the aggregate sum of the two will be relative to IRX $-\beta$. We constructed this figure by simulating $10^{6}$ pairs of components, where each indivdual component sits on the IRX- $\beta$ relation. To explain a sample of very blue DSFGs systematically offset from IRX $-\beta$ using this geometrical argument, the vast majority of DSFGs would need to be comprised of two radially different components (where IRX of the first would be a factor of $\gtrsim 100-1000$ times larger than the second). In other words, the distributions of IRX and $\beta$ over the spatial extent of a DSFG would need to be bimodal. Indeed, this is effectively what we see locally (e.g., Arp 220, Mrk 273, IRAS 19254-7245). The subcomponents of local ULIRGs that are infrared-bright produce very little UV light and the patches that are UV-bright are blue and see little IR contribution.

Aside from physical disassociation, alternative explanations also exist, although with less observational backing among local analogs. Nevertheless, we discuss them here since the effect we observe could be due in part to these multiple factors. As described in Section 4, Kong et al. (2004) suggest that IRX- $\beta$ should not, in fact, be a fixed relation for all galaxies, but rather it should vary for galaxies with different star formation histories. The more recent and intense the star formation, the bluer the galaxy (for a fixed $L_{\mathrm{IR}} / L_{\mathrm{UV}}$ ratio). While overall this interpretation explains Figure 5 qualitatively, we note that such a pronounced "break" above $L_{\mathrm{IR}} \approx 10^{11.5} L_{\odot}$ might not be expected. Whether or not it should be expected depends significantly on the given model assumptions for the effective absorption curve, and whether or not stars irradiate just their birth clouds or the ambient ISM (suggested to transition at a stellar age of $10 \mathrm{Myr}$; Charlot \& Fall 2000), as well as the distribution of the stars with respect to the interstellar medium (ISM), the lifetime of burst episodes (as short as $10 \mathrm{Myr}$ to as long as $300 \mathrm{Myr}$ ), and the characteristics of the underlying longterm star formation. Kong et al. (2004) provide a sensible model construct for quiescent and normal galaxies, but do not offer an explanation for sources at low $\beta$ and high IRX. Those galaxies could be caught during a very short duration burst, where a substantial fraction of the emitted UV radiation is trapped in stars' birth clouds before they have time to migrate out.

In addition to having a much bluer intrinsic UV slope, DSFGs might have enhanced $L_{\mathrm{IR}} / L_{\mathrm{UV}}$ ratios due to the short timescale of recent star formation. This might be due to UV radiation being trapped in stars' birth clouds or, more globally, could be explained by the geometry of dust and stars in the galaxy. In a well-mixed ISM with a more spheroidal distribution, the surface-to-volume ratio is lower, and a galaxy with higher SFR will have a higher $L_{\mathrm{IR}} / L_{\mathrm{UV}}$ ratio, assuming $L_{\mathrm{IR}}$ emanates from the whole volume while $L_{\mathrm{UV}}$ emerges only from regions close to the surface. On the contrary, lower luminosity, continuous-SFR disk galaxies have lower optical depth for dust attenuation and greater surface area-to-volume ratios, implying (1) $L_{\mathrm{IR}}$ and $L_{\mathrm{UV}}$ emanate from the whole volume, and (2) the underlying UV continuum is redder than in extreme burst galaxies.

Whether or not the magnitude of the shift toward bluer $\beta$ (or higher IRX) for DSFGs is in line with expectation requires more detailed model investigation, which is beyond the scope of this paper. Nevertheless, our results support the notion that DSFGs are likely inconsistent with prolonged, constant star formation histories of disk galaxies, as might be suggested by works favoring the galaxy main-sequence view of DSFGs at high $z$.

In the context of the galaxy main-sequence, readers might be curious how our results would present themselves if, instead of a $L_{\mathrm{IR}^{-}}$or SFR-dependent break in $\Delta \beta$, we investigated the break as a function of galaxy stellar mass or specific SFR. We 
have intentionally avoided the use of stellar mass estimates in this paper, primarily due to their reliance on an assumed star formation history. Infrared-luminous galaxies' star formation histories are particularly difficult to determine and have large uncertainties (Hainline et al. 2011; Michałowski et al. 2012, 2014). This in itself is a topic of ongoing debate in the literature (for further details, see Section 5 of Casey et al. 2014). We hope that follow-up studies that do investigate IRX- $\beta$ with stellar mass will carefully disentangle various star formation history a priori assumptions from any conclusions used to interpret galaxy evolution.

Delving deeper into the underlying physics behind bluer UV slopes, aside from our simple and favored geometric explanation, the possibility was raised in Section 4 that metallicity and possible top-heavy IMFs might also contribute to a higher proportion of $\mathrm{O}$ starlight and thus bluer slopes. A top-heavy IMF might be plausible in the dense star forming regions in ultraluminous galaxies, e.g., DSFGs, consistent with dense star clusters in the Milky-Way-like Arches and Westerlund 1 (Marks et al. 2012). Furthermore, our observation of some evolution in DSFG UV slopes of matched IR luminosity in the range $0.6<z<1.4$ would be consistent with the idea that higher-redshift systems are lower-metallicity systems. Note that if we assume that DSFGs at higher redshifts should be less bursty (as many papers suggest despite direct evidence to the contrary, e.g., Engel et al. 2010; Ivison et al. 2012), we might expect a shift in the opposite direction, toward redder UV slopes, or no shift at all. This is because main sequence galaxies are proposed to have steady state, approximately constant SFRs for most of their lifetime; even at very high SFRs ( $\gtrsim 100 M_{\odot} \mathrm{yr}^{-1}$ ), the expected underlying (unextincted) UV continuum of steady state star formation will be redder than starburst galaxies, which have a disproportionately large contribution of light from $\mathrm{O}$ and $\mathrm{B}$ stars above the less luminous and older A stars. Again, our results are inconsistent with the suggestion that $>10^{11.5} L_{\odot}$ DSFGs at high $z$ are dominated by steady state, secular, disky star formation.

\section{CONCLUSIONS}

This paper has investigated the relationship between the $L_{\mathrm{IR}} / L_{\mathrm{UV}}$ ratio (probing the relative "dustiness" of galaxies) to their rest-frame ultraviolet continuum slope, $\beta$, particularly as it pertains to infrared-selected galaxies. By comparing a sample of $\approx 1200$ nearby galaxies $(z<0.085)$ spanning SFRs 0.03-300 $M_{\odot} \mathrm{yr}^{-1}$ to a large sample of $\approx 4000$ IR-selected galaxies spanning photometric redshifts $0<z<5$ in the COSMOS field, we have arrived at the following conclusions.

1. We derive a much redder IRX $-\beta$ relationship for local galaxies than was presented in some of the original works on the topic (e.g., Meurer et al. 1999) and attribute the difference to (1) differences between $I U E$ and GALEX aperture limitations (the latter provides a more representative characteristic estimate for $\beta$ ), and (2) the fact that we derive the relation for a more heterogeneous population, not limited to galaxies selected as blue compact starbursts. Our derived relation is given in Equation (2). This is roughly consistent with prior works, which corrected the original relation for aperture differences (Takeuchi et al. 2012).

2. We find that at both low and high redshift, DSFGs with high SFRs above $\approx 50 M_{\odot} \mathrm{yr}^{-1}$ deviate from this IRX- $\beta$ relation toward bluer colors, where the offset grows with increasing IR luminosity and SFR. The deviation toward bluer colors, measured as $\Delta \beta$, is seen both in local and high- $z$ samples above a "break" IR luminosity of $\approx 10^{11-11.5} L_{\odot}$ and increases with increasing luminosity such that at $L_{\mathrm{IR}}=10^{13} L_{\odot}$, galaxies are on average $\Delta \beta=-2$ bluer than expected from the nominal IRX $-\beta$ relation fit in Equation (2). This offset toward bluer colors is shown not to be caused by sample selection effects.

3. Subtle redshift evolution is detected in the narrow luminosity regime $4-7.5 \times 10^{11} L_{\odot}$ for $0.6<z<1.4$, where galaxies of matched IR luminosity are on average $\Delta \beta=-1$ bluer at $z=1.4$ than at $z=0.6$. No redshift evolution is detected above $z>1.4$ (at representatively higher IR luminosities corresponding to detection limits at that epoch). More extensive samples of equal luminosity over a wider range of epochs are needed to verify this perceived evolution.

4. We attribute the bluer colors in dusty galaxies to more recent, rapid episodes of star formation (following the "birthrate" parameter model offered by Kong et al. 2004), where more IR luminous galaxies have a more prominent population of young, $\mathrm{O}$, and $\mathrm{B}$ stars (contributing to the rest-frame far-UV emission) than galaxies of more modest SFRs. Not only are they intrinsically bluer, DSFGs likely have higher IRX values caused by lower emergent UVto-IR luminosity ratios and mixed, patchy geometry. This is consistent with the idea that star formation in DSFGs at high redshift is dominated by burst activity rather than steady state, gradual disk growth.

5. With deep multi-band UV/optical and near-IR data in hand, we investigated the rates at which DSFGs contaminate high- $z$ LBG dropout searches. Due to the relatively low sky density of DSFGs, we find very low contamination rates at $z \lesssim 7$ when both deep optical and near-infrared data exist. Contamination rates increase at higher redshifts, up to $5.1 \%$ at $z \sim 10$, where there is less information on the rest-frame optical and more potential low-redshift interlopers (likewise, we see high contamination, $8.9 \%$, at $z \sim 6$ if observed near-infrared bands are not used for LBG selection). Overall, DSFG contamination rates $<10 \%$ bode well for LBG searches; however, we caution that this does not imply that $z>4$ SFRD estimates from LBGs alone are sufficient for understanding star formation at early times.

While this work has shed light on the issue of rest-frame ultraviolet emission in the dustiest galaxies, it is clear that much more work is necessary to understand the underlying physics driving these bluer rest-frame UV slopes in dusty galaxies. Geometry, morphological effects, and galaxy interactions need to be constrained on individual systems. Constraining these galaxies' star formation histories via detailed SED fitting is crucial to isolate the dominant physical mechanisms in high- $z$ star formation. The coming years will provide crucial insight into the issue of extinction in extreme galaxies by studying its links to gas dynamics, star formation rate, galaxy morphology, gas supply, dust reservoir, metallicity, and the differences between emission line and continuum extinction. This paper has only provided a broad context from which necessary followup studies of detailed systems will reveal the nature of dust attenuation in extreme environments.

COSMOS is based on observations with the NASA/ESA Hubble Space Telescope, obtained at the Space Telescope Science Institute, which is operated by AURA Inc, under NASA contract NAS 5-26555; also based on data collected at: the Subaru Telescope, which is operated by the National 
Astronomical Observatory of Japan; XMM-Newton, an ESA science mission with instruments and contributions directly funded by ESA Member States and NASA; the European Southern Observatory, Chile; Kitt Peak National Observatory, Cerro Tololo Inter-American Observatory, and the National Optical Astronomy Observatory, which are operated by the Association of Universities for Research in Astronomy, Inc. (AURA) under cooperative agreement with the National Science Foundation; the National Radio Astronomy Observatory, which is a facility of the National Science Foundation operated under cooperative agreement by Associated Universities, Inc; and the Canada-France-Hawaii Telescope operated by the National Research Council of Canada, the Centre National de la Recherche Scientifique de France, and the University of Hawaii.

PACS has been developed by a consortium of institutes led by MPE (Germany) and including UVIE (Austria); KU Leuven, CSL, IMEC (Belgium); CEA, LAM (France); MPIA (Germany); INAF-IFSI/OAA/OAP/OAT, LENS, SISSA (Italy); IAC (Spain). This development has been supported by the funding agencies BMVIT (Austria), ESA-PRODEX (Belgium), CEA/CNES (France), DLR (Germany), ASI/INAF (Italy), and CICYT/MCYT (Spain).

SPIRE has been developed by a consortium of institutes led by Cardiff Univ. (UK) and including: Univ. Lethbridge (Canada); NAOC (China); CEA, LAM (France); IFSI, Univ. Padua (Italy); IAC (Spain); Stockholm Observatory (Sweden); Imperial College London, RAL, UCL-MSSL, UKATC, Univ. Sussex (UK); and Caltech, JPL, HNSC, Univ. Colorado (USA). This development has been supported by national funding agencies: CSA (Canada); NAOC (China); CEA, CNES, CNRS (France); ASI (Italy); MCINN (Spain); SNSB (Sweden); STFC, UKSA (UK); and NASA (USA).

This research has made use of data from the HerMES project (http://hermes.sussex.ac.uk/). HerMES is a Herschel Key Programme utilizing Guaranteed Time from the SPIRE instrument team, ESAC scientists and a mission scientist. The data presented in this paper will be released through the HerMES Database in Marseille, HeDaM (http://hedam.oamp.fr/ HerMES/). We would also like to recognize the use of the Glue Visualization tool (www.glueviz.org) in the initial analysis of this data set.

C.M.C. acknowledges support from a McCue Fellowship through the University of California, Irvine's Center for Cosmology. A.C. acknowledges support from NSF AST-1313319. R.J.I. acknowledges support from ERC AdG, COSMICISM. T.T.T. has been supported by the Grant-in-Aid for the Scientific Research Fund (24111707) commissioned by the Ministry of Education, Culture, Sports, Science, and Technology (MEXT) of Japan. T.T.T. has also been partially supported from Strategic Young Researches Overseas Visits Program for Accelerating Brain Circulation from the MEXT. We are grateful to the anonymous referee for productive comments that improved the paper. We would also like to thank Naveen Reddy, Brian Siana, Mara Salvato, and Douglas Scott for helpful conversations during the manuscript's preparation.

\section{REFERENCES}

Adelberger, K. L., \& Steidel, C. C. 2000, ApJ, 544, 218

Alavi, A., Siana, B., Richard, J., et al. 2014, ApJ, 780, 143

Aretxaga, I., Wilson, G. W., Aguilar, E., et al. 2011, MNRAS, 415, 3831

Armus, L., Mazzarella, J. M., Evans, A. S., et al. 2009, PASP, 121, 559

Bauer, A. E., Conselice, C. J., Pérez-González, P. G., et al. 2011, MNRAS, 417, 289

Beckwith, S. V. W., Stiavelli, M., Koekemoer, A. M., et al. 2006, AJ, 132, 1729
Blain, A. W., Chapman, S. C., Smail, I., \& Ivison, R. 2004, ApJ, 611, 725 Boissier, S., Gil de Paz, A., Boselli, A., et al. 2007, ApJS, 173, 524 Boquien, M., Buat, V., Boselli, A., et al. 2012, A\&A, 539, A145

Boquien, M., Calzetti, D., Kennicutt, R., et al. 2009, ApJ, 706, 553 Bouwens, R. J., Illingworth, G. D., Franx, M., et al. 2009, ApJ, 705, 936 Bouwens, R. J., Illingworth, G. D., Labbe, I., et al. 2011a, Natur, 469, 504 Bouwens, R. J., Illingworth, G. D., Oesch, P. A., et al. 2011b, ApJ, 737, 90 Bouwens, R. J., Illingworth, G. D., Oesch, P. A., et al. 2014, arXiv:1403.4295 Brusa, M., Civano, F., Comastri, A., et al. 2010, ApJ, 716, 348 Bruzual, G., \& Charlot, S. 2003, MNRAS, 344, 1000

Buat, V., Giovannoli, E., Burgarella, D., et al. 2010, MNRAS, 409, L1 Buat, V., Giovannoli, E., Heinis, S., et al. 2011, A\&A, 533, A93 Buat, V., Iglesias-Páramo, J., Seibert, M., et al. 2005, ApJL, 619, L51 Buat, V., Noll, S., Burgarella, D., et al. 2012, A\&A, 545, A141 Bunker, A. J., Stanway, E. R., Ellis, R. S., McMahon, R. G., \& McCarthy, P. J. 2003, MNRAS, 342, L47

Burgarella, D., Buat, V., \& Iglesias-Páramo, J. 2005, MNRAS, 360, 1413 Calzetti, D. 2001, PASP, 113, 1449

Calzetti, D., Kinney, A. L., \& Storchi-Bergmann, T. 1994, ApJ, 429, 582 Capak, P., Aussel, H., Ajiki, M., et al. 2007, ApJS, 172, 99

Caputi, K. I., Lagache, G., Yan, L., et al. 2007, ApJ, 660, 97 Cardelli, J. A., Clayton, G. C., \& Mathis, J. S. 1989, ApJ, 345, 245 Casey, C. M. 2012, MNRAS, 425, 3094

Casey, C. M., Berta, S., Béthermin, M., et al. 2012a, ApJ, 761, 139 Casey, C. M., Berta, S., Béthermin, M., et al. 2012b, ApJ, 761, 140 Casey, C. M., Chapman, S. C., Beswick, R. J., et al. 2009, MNRAS, 399, 121 Casey, C. M., Chen, C.-C., Cowie, L. L., et al. 2013, MNRAS, 436, 1919 Casey, C. M., Narayanan, D., \& Cooray, A. 2014, PhR, 541, 45 Castellano, M., Sommariva, V., Fontana, A., et al. 2014, A\&A, 566, 19 Chapman, S. C., Blain, A. W., Smail, I., \& Ivison, R. J. 2005, ApJ, 622, 772 Chapman, S. C., \& Casey, C. M. 2009, MNRAS, 398, 1615

Chapman, S. C., Smail, I., Blain, A. W., \& Ivison, R. J. 2004a, ApJ, 614, 671 Chapman, S. C., Smail, I., Windhorst, R., Muxlow, T., \& Ivison, R. J. 2004b, ApJ, 611,732

Charlot, S., \& Fall, S. M. 2000, ApJ, 539, 718

Chary, R., \& Elbaz, D. 2001, ApJ, 556, 562

Civano, F., Elvis, M., Brusa, M., et al. 2012, ApJS, 201, 30

Conroy, C., Schiminovich, D., \& Blanton, M. R. 2010, ApJ, 718, 184

Cooray, A., Calanog, J., Wardlow, J. L., et al. 2014, ApJ, 790, 40

Coppin, K. E. K., Geach, J. E., Almaini, O., et al. 2014, arXiv:1407.6712

Cortese, L., Boselli, A., Buat, V., et al. 2006, ApJ, 637, 242

Dale, D. A., \& Helou, G. 2002, ApJ, 576, 159

Dey, A., Soifer, B. T., Desai, V., et al. 2008, ApJ, 677, 943

Donley, J. L., Koekemoer, A. M., Brusa, M., et al. 2012, ApJ, 748, 142

Dowell, C. D., Conley, A., Glenn, J., et al. 2014, ApJ, 780, 75

Dunne, L., Eales, S., Ivison, R., Morgan, H., \& Edmunds, M. 2003, Natur, 424, 285

Elbaz, D., Dickinson, M., Hwang, H. S., et al. 2011, A\&A, 533, A119

Engel, H., Tacconi, L. J., Davies, R. I., et al. 2010, ApJ, 724, 233

Finkelstein, S. L., Papovich, C., Giavalisco, M., et al. 2010, ApJ, 719, 1250

Finkelstein, S. L., Papovich, C., Salmon, B., et al. 2012, ApJ, 756, 164

Frayer, D. T., Smail, I., Ivison, R. J., \& Scoville, N. Z. 2000, AJ, 120, 1668

Fu, H., Jullo, E., Cooray, A., et al. 2012, ApJ, 753, 134

Giavalisco, M., Dickinson, M., Ferguson, H. C., et al. 2004, ApJL, 600, L103

Gil de Paz, A., Boissier, S., Madore, B. F., et al. 2007, ApJS, 173, 185

Goldader, J. D., Meurer, G., Heckman, T. M., et al. 2002, ApJ, 568, 651

Gordon, K. D., Clayton, G. C., Misselt, K. A., Landolt, A. U., \& Wolff, M. J. 2003, ApJ, 594, 279

Gordon, K. D., Clayton, G. C., Witt, A. N., \& Misselt, K. A. 2000, ApJ, 533,236

Goto, T., Takagi, T., Matsuhara, H., et al. 2010, A\&A, 514, A6

Griffin, M. J., Abergel, A., Abreu, A., et al. 2010, A\&A, 518, L3 Hainline, L. J., Blain, A. W., Smail, I., et al. 2011, ApJ, 740, 96 Hao, C.-N., Kennicutt, R. C., Johnson, B. D., et al. 2011, ApJ, 741, 124 Hathi, N. P., Malhotra, S., \& Rhoads, J. E. 2008, ApJ, 673, 686 Heinis, S., Buat, V., Béthermin, M., et al. 2013, MNRAS, 429, 1113 Hinshaw, G., Weiland, J. L., Hill, R. S., et al. 2009, ApJS, 180, 225 Hodge, J. A., Carilli, C. L., Walter, F., et al. 2012, ApJ, 760, 11 Hopkins, A. M., \& Beacom, J. F. 2006, ApJ, 651, 142 Howell, J. H., Armus, L., Mazzarella, J. M., et al. 2010, ApJ, 715, 572 Ilbert, O., Capak, P., Salvato, M., et al. 2009, ApJ, 690, 1236 Ilbert, O., Salvato, M., Le Floc'h, E., et al. 2010, ApJ, 709, 644 Ivison, R. J., Papadopoulos, P. P., Smail, I., et al. 2011, MNRAS, 412, 1913 Ivison, R. J., Smail, I., Amblard, A., et al. 2012, MNRAS, 425, 1320 Ivison, R. J., Smail, I., Le Borgne, J., et al. 1998, MNRAS, 298, 583 Kawada, M., Baba, H., Barthel, P. D., et al. 2007, PASJ, 59, 389 
Kinney, A. L., Bohlin, R. C., Calzetti, D., Panagia, N., \& Wyse, R. F. G. 1993, ApJS, 86, 5

Kong, X., Charlot, S., Brinchmann, J., \& Fall, S. M. 2004, MNRAS, 349, 769

Kriek, M., \& Conroy, C. 2013, ApJL, 775, L16

Le Floc'h, E., Papovich, C., Dole, H., et al. 2005, ApJ, 632, 169

Lee, K.-S., Alberts, S., Atlee, D., et al. 2012, ApJL, 758, L31

Lee, N., Sanders, D. B., Casey, C. M., et al. 2013, ApJ, 778, 131

Lilly, S. J., Tresse, L., Hammer, F., Crampton, D., \& Le Fevre, O. 1995, ApJ, 455,108

Lutz, D., Poglitsch, A., Altieri, B., et al. 2011, A\&A, 532, 90

Madau, P., Ferguson, H. C., Dickinson, M. E., et al. 1996, MNRAS, 283, 1388

Magdis, G. E., Elbaz, D., Dickinson, M., et al. 2011, A\&A, 534, A15

Marks, M., Kroupa, P., Dabringhausen, J., \& Pawlowski, M. S. 2012, MNRAS, 422, 2246

McCracken, H. J., Milvang-Jensen, B., Dunlop, J., et al. 2012, A\&A, 544, A156

Meurer, G. R., Heckman, T. M., \& Calzetti, D. 1999, ApJ, 521, 64

Meurer, G. R., Heckman, T. M., Leitherer, C., et al. 1995, AJ, 110, 2665

Michałowski, M. J., Dunlop, J. S., Cirasuolo, M., et al. 2012, A\&A, 541, A85

Michałowski, M. J., Hayward, C. C., Dunlop, J. S., et al. 2014, arXiv:1405.2335

Morrissey, P., Conrow, T., Barlow, T. A., et al. 2007, ApJS, 173, 682

Muñoz-Mateos, J. C., Gil de Paz, A., Boissier, S., et al. 2009, ApJ, 701, 1965

Neugebauer, G., Habing, H. J., van Duinen, R., et al. 1984, ApJL, 278, L1

Nguyen, H. T., Schulz, B., Levenson, L., et al. 2010, A\&A, 518, L5

Noeske, K. G., Weiner, B. J., Faber, S. M., et al. 2007, ApJL, 660, L43

Nordon, R., Lutz, D., Genzel, R., et al. 2012, ApJ, 745, 182

Oesch, P. A., Bouwens, R. J., Illingworth, G. D., et al. 2010, ApJL, 709, L16

Oesch, P. A., Bouwens, R. J., Illingworth, G. D., et al. 2013, ApJ, 773, 75

Oliver, S. J., Bock, J., Altieri, B., et al. 2012, MNRAS, 424, 1614

Ouchi, M., Shimasaku, K., Okamura, S., et al. 2004, ApJ, 611, 660

Overzier, R. A., Heckman, T. M., Wang, J., et al. 2011, ApJL, 726, L7

Papovich, C., Moustakas, L. A., Dickinson, M., et al. 2006, ApJ, 640, 92

Peek, J. E. G., \& Graves, G. J. 2010, ApJ, 719, 415

Penner, K., Dickinson, M., Pope, A., et al. 2012, ApJ, 759, 28

Pilbratt, G. L., Riedinger, J. R., Passvogel, T., et al. 2010, A\&A, 518, L1

Poglitsch, A., Waelkens, C., Geis, N., et al. 2010, A\&A, 518, L2

Reddy, N., Dickinson, M., Elbaz, D., et al. 2012, ApJ, 744, 154
Reddy, N. A., Erb, D. K., Pettini, M., Steidel, C. C., \& Shapley, A. E. 2010, ApJ 712,1070

Reddy, N. A., \& Steidel, C. C. 2009, ApJ, 692, 778

Reddy, N. A., Steidel, C. C., Erb, D. K., Shapley, A. E., \& Pettini, M. 2006, ApJ, 653, 1004

Riechers, D. A., Bradford, C. M., Clements, D. L., et al. 2013, Natur, 496, 329

Rodighiero, G., Daddi, E., Baronchelli, I., et al. 2011, ApJL, 739, L40

Roseboom, I. G., Dunlop, J. S., Cirasuolo, M., et al. 2013, MNRAS, 436, 430

Roseboom, I. G., Ivison, R. J., Greve, T. R., et al. 2012, MNRAS, 419, 2758

Roseboom, I. G., Oliver, S. J., Kunz, M., et al. 2010, MNRAS, 409, 48

Salpeter, E. E. 1955, ApJ, 121, 161

Sanders, D. B., Mazzarella, J. M., Kim, D.-C., Surace, J. A., \& Soifer, B. T. 2003, AJ, 126, 1607

Schlegel, D. J., Finkbeiner, D. P., \& Davis, M. 1998, ApJ, 500, 525

Scott, K. S., Austermann, J. E., Perera, T. A., et al. 2008, MNRAS, 385, 2225

Scoville, N., Arnouts, S., Aussel, H., et al. 2013, ApJS, 206, 3

Scoville, N., Aussel, H., Brusa, M., et al. 2007, ApJS, 172, 1

Seibert, M., Martin, D. C., Heckman, T. M., et al. 2005, ApJL, 619, L55

Shapley, A. E., Steidel, C. C., Pettini, M., \& Adelberger, K. L. 2003, ApJ, 588,65

Smail, I., Chapman, S. C., Blain, A. W., \& Ivison, R. J. 2004, ApJ, 616, 71

Smail, I., Ivison, R. J., \& Blain, A. W. 1997, ApJL, 490, L5

Stanway, E. R., Bunker, A. J., Glazebrook, K., et al. 2007, MNRAS, 376, 727

Stanway, E. R., McMahon, R. G., \& Bunker, A. J. 2005, MNRAS, 359, 1184

Steidel, C. C., Giavalisco, M., Pettini, M., Dickinson, M., \& Adelberger, K. L. 1996, ApJL, 462, L17

Swinbank, M., Smipson, J. M., Small, I., et al. 2013, MNRAS, 438, 1267

Symeonidis, M., Vaccari, M., Berta, S., et al. 2013, MNRAS, 431, 2317

Takeuchi, T. T., Buat, V., Heinis, S., et al. 2010, A\&A, 514, A4

Takeuchi, T. T., Yuan, F.-T., Ikeyama, A., Murata, K. L., \& Inoue, A. K 2012, ApJ, 755, 144

Tinsley, B. M., \& Danly, L. 1980, ApJ, 242, 435

To, C.-H., Wang, W.-H., \& Owen, F. N. 2014, ApJ, 792, 139

Trentham, N., Kormendy, J., \& Sanders, D. B. 1999, AJ, 117, 2152

U, V., Sanders, D. B., Mazzarella, J. M., et al. 2012, ApJS, 203, 9

Walter, F., Decarli, R., Carilli, C., et al. 2012, Natur, 486, 233

Wild, V., Charlot, S., Brinchmann, J., et al. 2011, MNRAS, 417, 1760 